\title{
Short Blankets in Snow Leopard Conservation: The Case of Qilianshan National Park (China) and Surrounding Areas
}

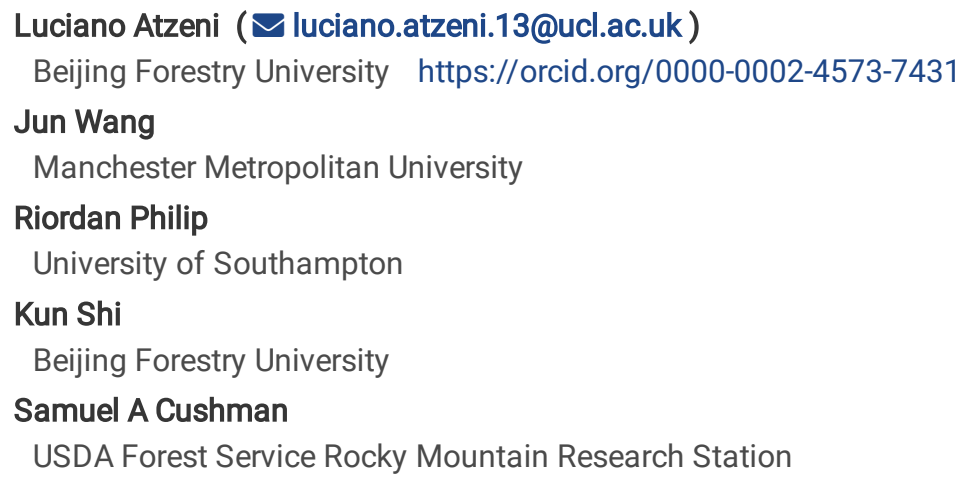

\section{Research Article}

Keywords: Landscape Connectivity, Resistant Kernel, Least-cost Path, UNICOR, Conefor, Protected Areas, Snow Leopard

Posted Date: March 3rd, 2022

DOI: https://doi.org/10.21203/rs.3.rs-1392903/v1

License: (9) This work is licensed under a Creative Commons Attribution 4.0 International License. Read Full License 


\section{Abstract}

\section{Context}

The assessment of landscape-level connectivity patterns is centrally important to the conservation management of wildlife species. This is especially true for highly vagile carnivore species, for which conservation efforts should extend beyond protected areas and link core populations.

\section{Objectives}

We investigated the effectiveness of the Qilianshan National Park (China), across the provinces of Gansu and Qinghai, in providing adequate conservation to snow leopard habitat and major dispersal routes.

\section{Methods}

We coupled resistant kernel and factorial least-cost path analyses to identify core home-range areas and major corridors connecting them. We compared two resistance surfaces derived from habitat and genetic models in their ability to describe connectivity routes. We ranked core areas and corridors based on their intensity of snow leopard movement flow.

\section{Results}

Much of the extent of core habitat patches, and a vast proportion of corridors length and area, were located outside the National Park. The kernel analyses identified a critical area to range-wide connectivity connected to the park but not formally protected. We identified important patches representing the backbone of snow leopard habitat and stepping-stone patches ensuring landscape structural and functional connectivity.

\section{Conclusions}

The Qilianshan National Park effectively protects some core snow leopard habitats. However, it alone appears insufficient to cover several important core areas and a number of critical connectivity linkages. In the context of ensuring meta-population persistence on the Tibetan Plateau and facilitate gene flow, conservation efforts should expand institute new protected areas strategically located to conserve core habitat areas and provide connectivity linkages among them.

\section{Introduction}

Landscape connectivity has been defined as the degree to which landscape features facilitate or impede movements of species among resource patches (Taylor et al. 1993). Preserving connectivity across landscapes ensures metapopulation persistence and population viability (Wiens 2001; Fahrig 2003) and is centrally important to successful conservation plans (Rudnick et al. 2012; Cushman et al. 2013a). Enhanced connectivity counterbalances risks posed by demographic fluctuations, habitat loss, fragmentation and population isolation (Rudnick et al. 2012; Cushman et al. 2013a). Fragmented and disconnected landscapes pose great threats to populations persistence, as they affect fitness success and increase mortality rates, reduce population sizes and prevent immigration and/or recolonization of areas after local extinction (Cushman et al. 2012a, 2013a; Kaszta et al. 2019, 2020a, b).

The effects of landscapes on animal movements are species-specific processes (Goodwin, 2003; Cushman, 2006). Functional connectivity, in fact, expresses the efficiency through which organisms move through different landscape structural characteristics (Rudnick et al. 2012; Zeller et al. 2012), and this ability depends on how the structure of the landscape resist movements, on local population abundances and on species-specific dispersal abilities (Cushman 2006; Zeller et al. 2012; Cushman et al. 2012a).

Each species will experience and perceive the landscape as a gradient of elements characterized by different 'permeability (Spear et al. 2010), the effects of which will vary greatly in the same context for different species, and for the same species in different contexts (Cushman et al. 2011, 2012b, 2013b). The accurate estimation of a resistance surface is the current basis for most assessments of functional connectivity, and its definition, evaluation, and when possible, validation, are critical steps in conservation planning (Spear et al. 2010; Zeller et al. 2012; Elliot et al. 2014; Cushman and Lewis 2010; Cushman et al. 2014).

A wide variety of approaches can be used to estimate how landscape characteristics resist movement. Ideally, direct estimates of animal dispersal based on movement data often provide the most robust understanding of species movement patterns (e.g., Elliot et al. 2014, Cushman and Lewis 2010; Cushman et al. 2014; Zeller et al. 2017, 2018). Since the collection of such information can be difficult (Zeller et al. 2012), other methods are also used, most often based on habitat model surrogates and landscape genetics (e.g., Khosravi et al. 2018; 
Macdonald et al. 2018; Cushman et al. 2014; Mateo-Sanchez et al. 2015a, b; Shirk et al. 2015). Among these, landscape genetics has been shown to often provide highly rigorous and reliable estimates of landscape resistance (e.g., Cushman et al. 2006; Wasserman et al. 2010; Mateo-Sanchez et al. 2015b; Shirk et al. 2015; Zeller et al. 2017), as it directly assesses landscape effects on functional connectivity itself, as measured by gene flow and genetic differentiation.

The use of habitat model surrogates would imply that the same factors affecting home range selection intervene also to influence movements related to mating and migration, which are two different biological processes (Cushman et al. 2013a). Recently, it has been shown that adopting an exponential transformation of habitat models might generate more realistic resistance surfaces, as this kind of transformations allow for dispersal in low-quality habitat, while an inverse transformation would constrain movement only in areas of high suitability (Zeller et al. 2018; Keeley et al. 2016, 2017; Mateo-Sanchez et al. 2015b). Since it is difficult to empirically validate habitat-derived resistance surfaces (Zeller et al. 2018), and given that habitat selection and movement might or might not rely on the same landscape constraints (Wasserman et al. 2010; Zeller et al. 2017; Cushman and Lewis 2010), resistance derived from suitability models are considered less accurate than those obtained using empirical genetic data (Zeller et al. 2018; Cushman and Lewis 2010; Wasserman et al. 2010). In such analyses, pairwise inter-individual measures of spatial genetic structure are employed to estimate functional connectivity as these reflect multi-generational dispersal events conducive of gene flow (Spear et al. 2010; Cushman et al. 2006).

Connectivity is a route-specific process (Cushman et al. 2009) and it must be evaluated with respects to movement paths across the resistance surface (Rudnick et al. 2012; Cushman et al. 2013a). Resistance surfaces per se, do not provide enough information on connectivity if not coupled with algorithms able to model animal movements (Diniz et al. 2020; Cushman et al. 2013a). Commonly, the least-cost path (LCP) (Dijkstra 1959; Adriansen et al. 2003), and the circuit theory (CT) algorithms are employed (McRae et al. 2008). These two approaches represent opposite views on animals' perception of landscapes, as the former assumes that individuals have perfect knowledge of the landscape to follow precisely the 'cheapest' route, while the latter assumes that all the possible paths on a landscape contribute to movement among a priori destinations (Spear et al. 2010; Cushman et al. 2013a).

When connectivity and corridors need to be estimated among hundreds or thousands of source-destination points, other methods provide a synoptic representation of landscape connectivity (sensu Cushman et al. 2014). The factorial LCP approach (Cushman et al. 2009), allows the calculation of LCP among all pairwise combinations of source-destination points within a specified dispersal distance threshold, emphasising the cumulative strength of routes in areas of the landscapes crossed by several LCPs (Cushman et al. 2009, Landguth et al. 2012). Another synoptic connectivity method, that complements factorial LCP analysis, is the resistant kernel approach (Compton et al. 2007). Resistant kernel analysis calculates the incidence function of expected movement density in all pixels of the landscape, based on the expected density of dispersers, the dispersal ability of the organism and the resistance of the landscape (Diniz et al. 2020; Cushman et al.

2013a). These two methods have synergistic use in conservation planning, as it is possible to identify, based on the strength of the resistant kernels, core areas for animals dispersal (Cushman et al 2013b, Cushman et al. 2018; Khosravi et al. 2018; Kaszta et al. 2020a), the importance of such areas within a network topology based on habitat availability index (Khosravi et al. 2018; Mohammadi et al. 2020), and the importance of factorial LCPs connecting such areas (Kaszta et al. 2020a; Macdonald et al. 2018; Cushman et al. 2018). This information combined ensures the definition of tailored and rigorous conservation initiatives to prioritise protection of both key core patches and dispersal corridors (Rudnick et al. 2012; Cushman et al. 2013a, b, 2014; Kaszta et al. 2020; MacDonald et al. 2018).

Furthermore, these methods are particularly useful when assessing the importance of protected areas (PAs) or PAs networks in ensuring an adequate degree of protection for a focal species (e.g., Kaszta et al. 2020; Mohammadi et al. 2020) or an array of species of conservation concern (Ashrafzadeh et al. 2020; Khosravi et al. 2020; Cushman et al. 2012), and in identifying and prioritizing areas for additional protection. Most often, existing PAs alone cannot guarantee the long-term persistence of populations, especially those characterised by large dispersal abilities and large home-range requirements (Cushman et al. 2013b; Diniz et al. 2017; Khosravi et al. 2018; Kaszta et al. 2020a; Ashrafzadeh et al. 2020; Johansson et al. 2016). While in certain instances PAs can efficiently preserve habitats (e.g., Moqanaki and Cushman 2017; Khosravi et al. 2018), they might not protect key dispersal routes (Ashrafzadeh et al. 2020; Cushman et al. 2018). Identifying both areas for persistence and dispersal, within and outside PAs, is particularly important for carnivore umbrella species. Given their habitat requirements and long travelled distances, the enhancement of protection of these areas can also offer wide benefits to many other species (Macdonald et al. 2020; Alexander et al. 2015).

The snow leopard (Panthera uncia) is widely regarded as the flagship species of central Asia, and a conservation ambassador for the mountain ecosystems in which it is found (McCarthy et al. 2017). Due to its elusiveness, snow leopard research is still characterized by many research gaps regarding population numbers (Suryawanshi et al. 2019), genetic diversity (Weckworth 2021) and connectivity (Riordan et al. 2016; Robinson and Weckworth 2016; Li et al. 2020). In this regard, there have been recent attempts to model both range-wide and local connectivity patterns. At a range level, Riordan et al. (2016) were the first to use an expert-based resistance surface to model resistant kernels across a range of potential dispersal thresholds, to highlight locations in the snow leopard range that could experience reduced

Page $3 / 24$ 
functional connectivity. Later, Li et al. (2020) integrated habitat modelling with circuit theory and least-cost path analysis to identify major linkages connecting landscape conservation units, derived upon a suitability surface. At a local level, Hameed et al. (2020) created a habitat model for the entirety of Pakistan, and used circuit theory to emphasise both the extent of suitable habitat and the intensity of connectivity pathways, discussing the effectiveness of Pakistan's PAs network to provide conservation both of habitat and dispersal routes. In Nepal, Shrestha and Kindlmann (2020) applied circuit theory to complement a genetic study, and identified potential connection areas linking genetically-verified snow leopard occurrences. Li et al. (2021) assessed the variations of snow leopard habitat under climate change scenarios in the Qinghai province of China, and identified stable habitat patches and corridors connecting them. Li et al. (2022) evaluated the impact of transportation infrastructures on snow leopard connectivity in the Qilianshan landscape, identifying important habitat and stepping stone patches, especially those not covered by protection status. With the exceptions of Li et al. (2020, 2021) the remaining studies did not adopt any framework for the identification, evaluation and prioritization of core habitat patches (sensu Cushman et al. 2013b) or key linkages (e.g., Kaszta et al. 2020a; Cushman et al. 2018). Gaining a robust and synoptic understanding of priority areas and dispersal routes is essential to establishing scientifically-based management actions (Cushman et al. 2018, Puyravaud et al. 2017; Kaszta et al. 2019, 2020a, b), that may greatly enhance inter-landscape connectivity and improve protection status.

In this study, we focus on the role of the Qilianshan National Park in representing a core protected area for snow leopard conservation at the north-eastern edge of the Qinghai-Tibetan Plateau. We apply resistant kernel analysis to calculate core habitat patches for snow leopard movement (within home ranges) and compare the resistance surfaces generated from habitat data (Atzeni et al. 2020) and from genetic data (Atzeni et al. in review) in their abilities to describe connectivity routes within and outside the national park. Atzeni et al. (2020) found that snow leopard probability of occurrence in this area was driven by the fine-scale alternation of ridges and valleys, by the degree of landscape aggregation, and by large extents of sparse vegetative conditions. In their landscape genetics analyses, Atzeni et al. (in review) used the genetic dataset described in Atzeni et al. (2021) to correlate genetic distances to landscape cost-distances relative to a series of predictors (and their combinations) hypothesized to have influence on snow leopard successful dispersal. They provided support for a model associating gene flow to flat areas at intermediate elevations, and low ruggedness conditions at a fine scale.

In particular, we seek to answer the following questions:

1) What is the importance of core habitat patches within QLSNP and adjoining areas?

2) What is the relative importance of core habitat patches within a network topology?

3) Which connectivity patterns will be inferred by the two resistance models?

4) What is the importance of linkages on the whole landscape?

5) What is the proportion of key linkages lying inside and outside QLSNP?

6) Which management recommendations can be inferred from the results?

\section{Methods}

\subsection{Study area}

The pilot area of Qilianshan National Park (hereafter QLSNP) (Figure 1) (N 36 $29^{\prime} 57^{\prime \prime} 39^{\circ} 43^{\prime} 39^{\prime \prime}, \mathrm{E}^{\circ} 7^{\circ} 23^{\prime} 34^{\prime \prime} 103^{\circ} 45^{\prime} 49^{\prime \prime}$ ), was established in 2018 and encompasses a total area of 50,200 km², distributed across Gansu Province (34,400 km²) and Qinghai Province (15,800 km²). It spans across several administrative jurisdictions at the prefecture and county levels (Figure 1). Topographically, it consists of a series of high mountains, valleys, and inter-mountain basins. Most of the elevations are above 3,000 - 3,500 meters, with an average altitude of approximately 4,000 meters. The annual average temperature in the pilot area is $-4{ }^{\circ} \mathrm{C}$, and characterized by a high temperature excursion, reaching $37.6{ }^{\circ} \mathrm{C}$ in summer and $-35.8^{\circ} \mathrm{C}$ in winter. The annual average precipitation amounts to $400 \mathrm{~mm}$. Vegetation types follow a clinal variation due to altitude gradients. These include mountain grassland (1,800 - 2,800 meters), temperate shrub steppe zone (2,000 - 2,200 meters), mountain forest grasslands (2,600 - 3,400 meters), subalpine shrub meadow zone (3,200 - 3,500 meters) and high mountain sparse vegetation zone (>3,500 meters). A consistent portion of the area is composed by meadow grassland and sparse vegetation, which cover more than $50 \%$ of the total area, dropping to $25 \%$ in the western region of the park, where alpine and desert grassland dominate, together with vegetation typical of swamp and saline meadows.

\subsection{Simulated points}


The best habitat suitability model for this area, from Atzeni et al. $(2020)$, was reclassified according to breaks corresponding to the $95^{\text {th }}, 85^{\text {th }}$ and $75^{\text {th }}$ percentiles of relative suitability values (Supplementary Figure S1). These represented high, medium-to-high, and low-to-high habitat thresholds, respectively. The amount of suitable habitat for each category has been calculated through a simple cell operation in ArcGIS, both relative to the total study area and to the extent delimited by QLSNP. The habitat categories and their areas will be reported for completeness, although they will be not processed further throughout the rest of this study.

In order to simulate connectivity and dispersal patterns in this landscape, we created a cloud of 100,000 random points on the whole extent. These points have been clipped to an area delimited by the patches encompassing medium-to-high quality habitat (8,528 points), representing the locations of the landscape where snow leopards are most likely to occur. From this subset, we selected five random draws of 1,000 points each to address location uncertainty and to evaluate the combined effect of different source/destination points.

Connectivity analyses described below were repeated on each of these five random draws of 1,000 points and the predictions were averaged to produce a spatially representative ensemble final prediction.

\subsection{Resistant Kernel Modelling}

The best suitability surface from Atzeni et al. (2020) was converted to a resistance layer using the exponential transformation $10^{(-1 . H S)}$

where HS represents the suitability scores. The resistance surface was then rescaled from 1 to 20 , using the raster.transformation tool in SpatialEco (Evans 2020), after its cell size was upscaled from 90 to 500 meters, to reduce the computational time (Figure 2). Upscaling to a coarse cell size has a negligible effect in connectivity analyses, provided important landscape characteristics are not lost due to excessive coarsening (McRae et al. 2008; Cushman and Landguth 2010).

UNICOR (Landguth et al. 2012) was used to calculate cumulative resistant kernels (Compton et al. 2007), at thresholds corresponding to 25, 50, 100 and 200 km-equivalent. These thresholds were chosen to approximate daily to home-range level movements (McCarthy et al. 2005; Johansson et al. 2018).

A threshold corresponding to the $85^{\text {th }}$ percentile of the averaged kernel surfaces was chosen to identify movement core areas for each kernel. Several authors have adopted this strategy to identify the fracture zones (sensu Cushman et al. 2013b) separating core movement probability patches (Cushman et al. 2013b; Cushman et al. 2018; Khosravi et al. 2018; Macdonald et al. 2018; Kaszta et al. 2020a; Ashfrazdeh et al. 2020). These core patches were then analysed in Fragstats (McGarigal et al. 2012) to calculate the following class-level metrics: largest patch index (LPI), number of patches (NP), percentage of landscape (PLAND), Class area (area-weighted mean) (AREA_AM), and class radius of gyration (area-weighted mean, also known as correlation length) (GYRATE_AM). At the patch-level, Area of each core patch was calculated. PLAND is the simplest metric of landscape composition, quantifying the proportion of the total area occupied by the patch of interest. NP is a simple measure of habitat subdivision, and reports the number of fragments in the landscape of a particular patch type. LPI describes the magnitude of the largest patch of a given type as proportion relative to the total landscape area (Cushman et al. 2013b, McGarigal et al. 2012). GYRATE_AM represents the average distance an animal can travel before leaving a patch when dropped at random on the landscape. AREA_AM expresses the average patch area conditions that the focal species would experience if dropped at random on the landscape. These metrics were selected based on past studies that showed they were particularly useful as measures of how landscape patterns affect connectivity processes (e.g., Wasserman et al. 2012a, b; Cushman et al. 2013c).

The same statistics have been calculated for the core areas identified within QLSNP. This had the purpose of quantifying the amount of core patches under protection status and that lying outside the national park, in contiguous areas belonging to snow leopard range, and functionally connected to the protected area. The importance of core patches (relative to the whole extent) was evaluated ranking them by their total area, multiplied by the sum of the kernel values (strength) contained within the patches (Kaszta et al. 2020a; Cushman et al. 2018).

\subsection{Contribution of core areas to landscape connectivity}

The software Conefor 2.6 (Saura and Torné 2009) was used to establish the relative importance of each of the core area patches within the topology representing total network connectivity. Conefor relies on a graph-structure representation of the landscape patterns (PascualHortal and Saura 2006), composed by a set of nodes and links, representing respectively patches of suitable habitat and functional connections between any two patches as a function of distance (Urban and Keitt 2001; Pascual-Hortal and Saura 2006). It quantifies habitat availability indices that consider simultaneously the connectivity within each patch and connectivity among patches, evaluating at the same time habitat abundance and rate of connectivity (Pascual-Hortal and Saura 2006). 
The strength of each core patch was used as node attribute in each graph analysis. Initially, topologies were evaluated at the same thresholds used for resistance kernel analyses, to account for different dispersal scales integrating daily movements and large distance dispersal abilities. However, since the importance of the patches did not vary, only results relative to the largest threshold (200 km) will be displayed. Moreover, only results relative to 25 and $50 \mathrm{~km}$ resistance kernel core patches (hereafter $\mathrm{K} 25$ and $\mathrm{K} 50$ ) will be included, as they possessed more heterogeneity, and the results from the largest kernels were redundant.

The importance of the core patches within the topologies was evaluated using the probability of connectivity index (PC) (Saura and Pascual-Hortal 2007), using the importance of core patches as the attribute field. As the index ranges 0-1, it was quantified through a relative ranking, calculating the percentage of importance of each topology element (Urban and Keitt 2001; Saura and Pascual-Hortal 2007; Pascual-Hortal and Saura 2006):

$$
d P C(\%)=\frac{P C-P C^{\prime}}{P C} \times 100
$$

where $P C^{\prime}$ is the index value after removal of a certain patch, and $P C$ is the value when that patch is present (Saura and Pascual-Hortal 2007). Therefore, dPC quantifies the importance of each landscape element for overall habitat availability with larger values indicating that removal of a particular element will interrupt the links between the other patches, since no patch is present in the topology that can substitute for it (Saura and Rubio 2010).

Later research using this metric showed that it can be decomposed into three different fractions, quantifying different connectivity attributes, as one drawback of dPC is the tendency to assign greater importance to larger patches (Ferrari et al. 2007), and does not specifically quantify the importance of a patch as a stepping stone element (Saura and Rubio 2010). Specifically, dPC can be decomposed in

$d P C=d P C_{\text {intra }}+d P C_{\text {flux }}+d P C_{\text {connector }}$

The fraction $\mathrm{dPC}_{\text {intra }}$ (hereafter Intra) measures exclusively intra-patch connectivity, and does not depend on how a patch is connected to others. The fraction $\mathrm{dPC}_{\text {flux }}$ (hereafter Flux) depends on both patch attribute and its position in the connection network, measuring an attribute-weighted dispersal flux through the connections of a given patch, when this is the starting or ending point of a connection. However, it does not measure the importance of each element for maintaining the connectivity between the other patches. The last fraction, $\mathrm{dPC}_{\text {connector }}$ (hereafter Connector), depends on the position of an element in the topology, is independent of the attributes, and measures the importance of a patch when it is part of the maximum product probability path for dispersal between any two other patches (Saura and Rubio 2010).

\subsection{Dispersal corridors}

The above analyses rely exclusively on edge-to-edge patch Euclidean distances, and as such, even identifying elements' importance within the connection network, do not provide information on the relative importance of links within the topology, and how these would vary as a function of different resistance models. In order to explore this issue, we adopted the factorial least-cost path approach (Cushman et al. 2009), implemented in UNICOR (Landguth et al. 2012). The best genetic resistance surface from Atzeni et al. (in review), was upscaled to match the extent of this study, at a cell size of 500 meters, and stretched 1-20 in spatialEco (Evans 2020), to allow full comparability with the resistance model derived from habitat suitability (Figure 2). Factorial least-cost paths were calculated for each of the five draws of points at a threshold of 2 million cost-units, to model multi-generational landscape-level dispersal pathways (e.g., Kaszta et al. 2020a). We smoothed the corridors using a width of $10 \mathrm{~km}$, and averaged the outputs across the five sets of occurrences. We computed Pearson's correlation between scenarios to quantify their agreement, using the Band Collection Statistics Tool in ArcGIS. Furthermore, the two scenarios were summed to provide a cumulative representation of all possible dispersal pathways.

We ranked the importance of the corridors connecting the core areas identified by $\mathrm{K} 25$ and $\mathrm{K} 50$. We reclassified the factorial least-cost path rasters from either scenario, and their summed output, using the $95^{\text {th }}$ percentile of the rasters value distribution. Then, we created corridors fragments isolating all paths lying outside the core habitat patches. The reclassified outputs were used to calculate LPI, NP, AREA_AM and GYRATE_AM at the class level, and AREA and GYRATE at the patch-level in Fragstats (McGarigal et al. 2012). The same metrics have been calculated for all the corridors delimited by the extent of the national park to quantify the magnitude of links lying outside its boundaries. The importance of each corridor (on the whole extent) was ranked using their radius of gyration (expressing the area-weighted average length of the pathways), multiplied by the sum of the least-cost path intensity values within each corridor (e.g.., Kaszta et al. 2019, 2020a). As a last weighting step, the corridors were multiplied by the average strength of the core kernel patches they intersected (Kaszta et al 
2020a; Cushman et al. 2018) within a $5 \mathrm{~km}$ radius to account for routes in close proximity to core areas, which is necessary to address location uncertainty in dispersal routes.

\section{Results}

\subsection{Habitat extent and resistant kernel core areas}

The re-categorization of the habitat surface from Atzeni et al. (2020) produced a total amount of suitable snow leopard habitat equal to $15,716 \mathrm{Km}^{2}$ (high quality), 41,148 $\mathrm{Km}^{2}$ (medium-to-high quality) and 78,580 $\mathrm{Km}^{2}$ (low-to-high quality) (Table 1 ). Following the same order, the proportion of habitat classes inside QLSNP amounted 6,767 $\mathrm{Km}^{2}$ (43.1\% of the total), $17,769 \mathrm{Km}^{2}$ (37.7\% of the total) and $27,010 \mathrm{Km}{ }^{2}$ (34.4\% of the total). The total area encompassed by resistant kernel core habitat patches did not vary across the dispersal thresholds (Table 2), amounting 47,154.5 $\mathrm{Km}^{2}$. Inside QLSNP, the proportion of core kernel areas relative to the total was $40.2 \%$ (25 $\mathrm{km}$ threshold), $39.7 \%$ (50 km threshold), 40.4\% (100 km threshold) and 42.6\% (200 km threshold) (Table 2).

Table 1

Extent of suitable habitat for the whole landscape analysed and inside Qilianshan National Park (QLSNP). Ratio refers to the proportion within QLSNP with respect to the total.

\begin{tabular}{|c|c|c|c|}
\hline \multirow[t]{2}{*}{ Habitat quality } & \multicolumn{2}{|c|}{ Extent in $\mathrm{Km}^{2}$} & \multirow[t]{2}{*}{ Ratio } \\
\hline & Whole & QLSNP & \\
\hline High & 15716 & 6767 & 0.431 \\
\hline Medium-to-high & 47148 & 17769 & 0.377 \\
\hline Low-to-high & 78580 & 27010 & 0.344 \\
\hline
\end{tabular}

Table 2

Descriptive statistics relative to the resistant kernel core areas, at all thresholds considered. PLAND = percentage of landscape; NP = number of patches; LPI = largest patch index; Gyrate_Am = Class area-weighted mean radius of gyration; Area_Am = Class area area-weighted mean. Total Area $=$ sum of the area of all patches.

\begin{tabular}{|c|c|c|c|c|c|}
\hline \multirow[b]{2}{*}{ Metrics } & & \multicolumn{4}{|c|}{ Resistant Kernel Core Patches } \\
\hline & & 25 km & 50 km & 100 km & 200 km \\
\hline \multirow[t]{2}{*}{ PLAND } & Total & 15 & 15 & 15 & 15 \\
\hline & QLSNP & 37.726 & 37.344 & 37.961 & 40.007 \\
\hline \multirow[t]{2}{*}{ NP } & Total & 92 & 21 & 11 & 4 \\
\hline & QLSNP & 64 & 26 & 17 & 7 \\
\hline \multirow[t]{2}{*}{ LPI } & Total & 3.610 & 6.567 & 7.313 & 7.786 \\
\hline & QLSNP & 12.968 & 15.324 & 17.264 & 20.190 \\
\hline \multirow[t]{3}{*}{ Gyrate_Am (km) } & Total & 41.109 & 63.066 & 65.963 & 64.010 \\
\hline & QLSNP & 34.879 & 37.822 & 38.192 & 40.270 \\
\hline & Park/Total & 0.848 & 0.600 & 0.579 & 0.629 \\
\hline \multirow[t]{3}{*}{ Area_Am $\left(\mathrm{km}^{2}\right)$} & Total & 5898.782 & 12603.235 & 15628.698 & 17947.223 \\
\hline & QLSNP & 3438.698 & 4534.328 & 5493.188 & 6887.208 \\
\hline & Park/Total & 0.583 & 0.360 & 0.351 & 0.384 \\
\hline \multirow[t]{3}{*}{ Total Area $\left(\mathrm{km}^{2}\right)$} & Total & 47154.5 & 47154.5 & 47154.5 & 47154.5 \\
\hline & QLSNP & 18935.5 & 18743.75 & 19053.25 & 20080.5 \\
\hline & Park/Total & 0.402 & 0.397 & 0.404 & 0.426 \\
\hline
\end{tabular}


Always inside QLSNP, the percentage of the landscape composed of core kernel patches (representing dispersal in medium-to-high habitat quality) amounted $37.72 \%, 37.34 \%, 37.96 \%$ and $40 \%$ for $25,50,100$, and $200 \mathrm{~km}$ dispersal, respectively (Table 2 ).

On the whole extent, we found 92 kernel core patches at $25 \mathrm{~km}$ dispersal, 21 at $50 \mathrm{~km}, 11$ at $100 \mathrm{~km}$ and only 4 at $200 \mathrm{~km}$. The number of patches inside QLSNP decreased substantially at the $25 \mathrm{~km}$ dispersal, while it increased at the remaining dispersal thresholds, likely because many large high density movement areas were split into more smaller parts after delimiting the kernel patches to the size of QLSNP (Table 2). On average, patches inside QLSNP were smaller (AREA_AM) and shorter (GYRATE_AM) than those calculated on the total extent (Table 2). Considering the average patch area conditions (AREA_AM), QLSNP accounted for $58.3,36,35.1$ and $38.4 \%$ of the average patch area (AREA_AM), compared to the total extent, for the 25, 50, 100, $200 \mathrm{~km}$ dispersal thresholds respectively (Table 2). Likewise, QLSNP accounted for $84.8(25 \mathrm{~km}), 60(50 \mathrm{~km}), 57.9(100 \mathrm{~km})$ and $62.9 \%(200 \mathrm{~km})$ of the average radius of gyration (GYRATE_AM) compared to the total (Table 2). Ranking of core area patches at all kernel thresholds is reported in Supplementary Tables S1 - S3, and visually displayed in Fig. 3, where the most important 25 and 20 patches are displayed for the resistant kernels at 25 and $50 \mathrm{~km}$ thresholds, respectively.

\subsection{Core Areas Importance To Network Connectivity}

Overall, patches that ranked high according to their kernel strength values and size (Supplementary Tables S1 - S3), were also those found to be more conducive of intra-patch movements, as expected (Fig. 4). The K25 patches found important core areas conducive of high intrapatch movement located inside QLSNP on the north-western part (Patch 1; 11,347.8 $\mathrm{Km}^{2}$ ), central part (Patch 4; 4,943 $\mathrm{Km}^{2}$ ) and southeastern part (Patch 3; 6,348.75 $\mathrm{Km}^{2}$ ). Another important patch was located outside QLSNP, in the counties of Tianjun and Delingha (Patch 2; $7,855 \mathrm{Km}^{2}$ ) (Fig. 4; Supplementary Table S1). Considering the dPC Flux and Connector fractions, the movement of individuals appeared continuous from patches 1 to 7 , thanks to their extensiveness and proximity, structurally connecting all major backbone patches among themselves. Patches 5 and 6 , across the counties of Tianjun, Gangca and Qilian, provided an important linkage between the central part of QLSNP and Patch 2. Patches 8 to 10 provided represented the most important stepping stone patches outside (Patches 8 and 9) and inside (patch 10) QLSNP. Patches 8 and 9, along with minor fragments, ensured continuity of flux and connections between the north-westernmost portion (Patch 7) and the central south portion (Patch 2) (Fig. 4; Supplementary Table S1).

The K50 patches described one uninterrupted large area of intra-patch movement (Patch 1; 20,644 Km²), spanning over Sunan Yugur, Qilian, Tianjun, Gangca and Delingha counties (Fig. 4; Supplementary Table S2). Other important patches for snow leopard persistence were found across Subei and Sunan Yugur (Patch 2; 10,347 Km²), Subei (Patch 4; 2,308 Km²), and stretching over many administrative units in the south-east (Patch $3 ; 7,174 \mathrm{Km}^{2}$ ). The dPC Flux and Connector fractions for this kernel threshold were slightly different (Fig. 4). The importance of dPC Flux fraction was consistent with dPC Intra for the largest patches, with patches 5 to 7 (over $800 \mathrm{Km}^{2}$; Supplementary Table S2) acting as important flux fragments in areas structurally connected to QLSNP, but outside or overlapping its boundaries. The dPC Connector fraction for this kernel threshold described how the integrity of the connections on the whole landscape is pillared by Patches 1 and 2, with Patches 4, 9, 12, and 13 ensuring the continuity of the movement between the north-western portion and the south-central part of the extent (Fig. 4; Supplementary Table S2).

\subsection{Factorial Least-cost Path Corridors And Patterns}

Overall, the number of corridor fragments connecting the K25 core patches was always higher than those connecting the K50 core patches, considering either resistance scenario and their cumulative output (Table 3). QLSNP accounted for $50 \%$ of corridors, considering the habitatbased resistance, 55 to $65 \%$ of corridors considering the genetic resistance and 53 to $58 \%$ considering all corridors from either scenario (Table 3). 
Table 3

Descriptive statistics relative to the factorial least-cost paths, for the resistance derived from habitat suitability (Habitat), the genetic resistance (Genetic) and their summed outputs (Cumulative). NP = number of patches; LPI = largest patch index; Gyrate_Am = Class areaweighted mean radius of gyration; Area_Am = Class area area-weighted mean. Total Gyrate $=$ sum of the radius of gyration of all patches; Total Area $=$ sum of the area of all patches. K25 = core area patches calculated from a resistant kernel at a dispersal threshold of $25 \mathrm{~km}-$ equivalent. $\mathrm{K} 50$ = core area patches calculated from a resistant kernel at a dispersal threshold of $50 \mathrm{~km}$-equivalent.

\begin{tabular}{|c|c|c|c|c|c|c|c|c|c|c|}
\hline \multirow[t]{2}{*}{ Metrics } & \multirow{2}{*}{$\begin{array}{l}\text { Core } \\
\text { patches }\end{array}$} & \multicolumn{3}{|c|}{ Whole extent } & \multicolumn{3}{|l|}{ QLSNP } & \multicolumn{3}{|c|}{ Ratio (Park/Total) } \\
\hline & & Habitat & Genetic & Cumulative & Habitat & Genetic & Cumulative & Habitat & Genetic & Cumulative \\
\hline \multirow[t]{2}{*}{ NP } & K25 & 234 & 107 & 173 & 117 & 59 & 92 & 0.50 & 0.55 & 0.53 \\
\hline & K50 & 153 & 60 & 109 & 77 & 39 & 63 & 0.50 & 0.65 & 0.58 \\
\hline \multirow[t]{2}{*}{ LPI } & K25 & 9.52 & 19.87 & 19.05 & 10.16 & 25.81 & 15.20 & \multirow{2}{*}{\multicolumn{3}{|c|}{ Not Applicable }} \\
\hline & K50 & 15.31 & 32.40 & 24.19 & 12.91 & 27.31 & 18.56 & & & \\
\hline \multirow{2}{*}{$\begin{array}{l}\text { Gyrate_Am } \\
(\mathrm{km})\end{array}$} & K25 & 10.49 & 51.40 & 39.77 & 5.72 & 14.06 & 10.70 & 0.55 & 0.27 & 0.27 \\
\hline & K50 & 14.55 & 64.32 & 46.67 & 9.00 & 17.08 & 13.19 & 0.62 & 0.27 & 0.28 \\
\hline \multirow{2}{*}{$\begin{array}{l}\text { Area_Am } \\
\left(\mathrm{km}^{2}\right)\end{array}$} & K25 & 224.86 & 1856.82 & 1088.43 & 100.01 & 439.79 & 218.68 & 0.44 & 0.24 & 0.20 \\
\hline & K50 & 351.73 & 2739.53 & 1519.99 & 156.61 & 565.57 & 285.22 & 0.45 & 0.21 & 0.19 \\
\hline \multirow{2}{*}{$\begin{array}{l}\text { Total } \\
\text { Gyrate } \\
(\mathrm{km})\end{array}$} & $\mathrm{K} 25$ & 509.36 & 544.81 & 589.07 & 207.83 & 188.08 & 218.70 & 0.41 & 0.35 & 0.37 \\
\hline & K50 & 435.33 & 447.15 & 488.67 & 193.71 & 174.88 & 201.64 & 0.44 & 0.39 & 0.41 \\
\hline \multirow{2}{*}{$\begin{array}{l}\text { Total Area } \\
\left(\mathrm{km}^{2}\right)\end{array}$} & K25 & 6538.00 & 13806.00 & 10578.00 & 2480.00 & 3782.50 & 3224.25 & 0.38 & 0.27 & 0.30 \\
\hline & K50 & 6587.75 & 13869.25 & 10539.25 & 2658.25 & 3999.25 & 3286.50 & 0.40 & 0.29 & 0.31 \\
\hline
\end{tabular}

The total length of corridors was lower for the K50 core patches compared to K25, considering all dispersal scenarios. QLSNP encompassed a low percentage of the corridor's total radius of gyration, ranging from $41-44 \%$ (habitat-based scenario), $35-39 \%$ (genetic scenario) and $37-41 \%$ (cumulative output) (Table 2 ).

On the whole extent, the genetic scenario produced $13,806 \mathrm{Km}^{2}$ and $13,869.25 \mathrm{Km}^{2}$ of corridors connecting $\mathrm{K} 25$ and $\mathrm{K} 50$ patches, respectively (Table 3). The habitat-based scenario retrieved instead 6,538 and 6,587.75 $\mathrm{Km}^{2}$ of corridors connecting $\mathrm{K} 25$ and $\mathrm{K} 50$ patches, respectively. Following the same order, the total corridor areas for the cumulative output were 10,578 and 10,539 Km². Inside QLSNP, the total area encompassed by corridors dropped considerably compared to the whole extent, representing $38-40 \%$ of the total (habitat-based scenario), $27-29 \%$ of the total (genetic scenario) and $30-31 \%$ of the total (cumulative output) (Table 3).

On average, links from the habitat-based scenario were shorter (GYRATE_AM) and smaller (AREA_AM) than those derived from the genetic scenario. The average corridor radius of gyration (GYRATE_AM) decreased substantially in the area delimited by QLSNP, representing 55$62 \%$ of the total (habitat-based), 27\% (genetic), and 27-28\% (cumulative). Likewise, the average corridor area (AREA_AM) was smaller inside QLSNP, compared to the total, amounting 44-45\% of the total average area (habitat-based), 21-24\% (genetic) and 19-20\% (cumulative) (Table 3).

Visually, the outputs of factorial least-cost path surfaces highlighted important differences between resistance models (Supplementary Figure S2), exemplified by the low correlation coefficient (Pearson's $r=0.083$ ). In general, the major factorial least-cost paths in the habitatbased scenario followed conditions strictly associated with habitat, along ridgelines and fine-scale topographic depressions in high mountain systems (Supplementary Figure S2). Conversely, the major factorial least-cost paths in the genetic scenario run through large flat open areas at the foot of mountain ranges (Supplementary Figure S2).

The 20 best corridors for each scenario of resistance have been identified and plotted (Fig. 5; Table 4). For the habitat-based resistance, considering both $\mathrm{K} 25$ and $\mathrm{K} 50$, the two most important corridors connected the two most important habitat patches located in the northern and southern portions. Notable links connected the south-eastern core areas to the major patches along the Qilian mountains both outside and inside the national park (links 3 and 4, respectively, for both K25 and K50). Additional corridors were generally found within the mountain areas and within the park, ensuring connectivity among smaller patches. Much of the inferred most important corridors (about half for both kernel types) were located in areas outside QLSNP (Fig. 5; Table 3). Considering the genetic resistance, the two most important paths directly connected the south-eastern end of the mountainous range to the north-western part through a series of links between 
stepping stone core areas of various size (Fig. 5). Link 3 for both kernel types connected simultaneously the north-western, the central and the southern core areas through multiple bifurcations, while links 4 and 5 steadily ensured continuity among habitat patches located on the north-western side (Fig. 5; Table 4). Pathways for the cumulative factorial least cost-paths encompassed all the major corridors from either scenario, whose final ranking was consistent with their importance in single scenarios (Fig. 6; Table 4; Supplementary Figure S3).

Table 4

Radius of gyration (in $\mathrm{Km}$ ) of the 20 most important factorial leastcost path corridors for the Genetic-based resistance, Habitat-based resistance, and the cumulative rasters encompassing both scenarios. $\mathrm{K} 25$ = core area patches calculated from a resistant kernel at a dispersal threshold of $25 \mathrm{~km}$-equivalent. $\mathrm{K} 50=$ core area patches calculated from a resistant kernel at a dispersal threshold of $50 \mathrm{~km}$ equivalent.

\begin{tabular}{|c|c|c|c|c|c|c|}
\hline & \multicolumn{6}{|c|}{ Models } \\
\hline & \multicolumn{2}{|c|}{ Genetic resistance } & \multicolumn{2}{|c|}{ Habitat resistance } & \multicolumn{2}{|c|}{ Cumulative } \\
\hline & \multicolumn{6}{|c|}{ Core areas } \\
\hline Rank & $K 25$ & $K 50$ & $K 25$ & $K 50$ & $K 25$ & $K 50$ \\
\hline 1 & 62.95 & 60.36 & 16.96 & 34.86 & 89.48 & 57.68 \\
\hline 2 & 99.91 & 99.42 & 23.08 & 18.72 & 59.30 & 88.84 \\
\hline 3 & 51.34 & 79.46 & 21.77 & 17.95 & 36.80 & 47.97 \\
\hline 4 & 44.05 & 44.24 & 10.47 & 9.28 & 20.25 & 26.40 \\
\hline 5 & 29.66 & 29.15 & 15.43 & 14.41 & 31.91 & 41.59 \\
\hline 6 & 24.86 & 18.36 & 16.01 & 6.54 & 27.30 & 26.69 \\
\hline 7 & 12.21 & 5.81 & 15.64 & 18.24 & 10.23 & 7.11 \\
\hline 8 & 20.04 & 3.30 & 11.34 & 8.25 & 14.87 & 5.61 \\
\hline 9 & 5.21 & 3.63 & 6.67 & 15.09 & 8.99 & 17.82 \\
\hline 10 & 3.25 & 10.50 & 7.20 & 4.51 & 19.87 & 8.11 \\
\hline 11 & 3.43 & 12.21 & 11.43 & 7.86 & 8.86 & 11.73 \\
\hline 12 & 2.18 & 2.93 & 6.50 & 11.83 & 5.94 & 7.16 \\
\hline 13 & 10.20 & 3.07 & 9.85 & 11.44 & 5.47 & 3.52 \\
\hline 14 & 12.28 & 3.45 & 5.32 & 12.49 & 8.01 & 5.82 \\
\hline 15 & 2.88 & 2.21 & 6.31 & 16.17 & 3.32 & 4.47 \\
\hline 16 & 4.26 & 2.33 & 3.17 & 2.74 & 7.23 & 3.54 \\
\hline 17 & 3.05 & 2.65 & 4.51 & 8.61 & 6.83 & 5.50 \\
\hline 18 & 2.69 & 2.45 & 2.84 & 3.75 & 2.82 & 3.42 \\
\hline 19 & 21.62 & 1.96 & 2.30 & 4.82 & 4.02 & 2.61 \\
\hline 20 & 2.74 & 1.64 & 7.19 & 5.72 & 3.40 & 2.36 \\
\hline
\end{tabular}

\section{Discussion}

This study complements a recent analysis from Li et al. (2022), which assessed suitable habitat and conservation gaps for the snow leopard in the Qilianshan landscape and surrounding areas, with emphasis on the role of QLSNP. Overall, our analysis found a bigger extent of suitable habitat than Li et al. (2022), but both studies tend to agree in their estimate of suitable snow leopard habitat inside QLSNP (Table 1). These discrepancies, as well as the patterns of the suitability maps (Atzeni et al. 2020; Li et al. 2022), come from different modelling approaches, since Li et al. (2022) did not implement model selection based on ecological scales or subset combinations of variables (as in Atzeni et al. 2020). Nevertheless, this study, along with previous research that dealt with the issue of snow leopard connectivity in this part of the range (Riordan et al. 2016; Li et al. 2020, 2021, 2022), provides further evidence on the need to integrate 
additional snow leopard habitat (either conducive of long-range movement or essential for species persistence) into conservation management plans. We will discuss the major findings, together with some technical considerations on the use of resistance maps, and provide advice to enhance the protection of snow leopards in China.

\subsection{Core areas importance}

This study identified core area patches at several levels of resistant kernel thresholds, using a habitat-derived surface as resistance scenario. Most commonly, habitat patches alone (Supplementary Figure S1) are not a sufficient proxy for population size (Cushman et al. 2009), movement patterns (Cushman et al. 2014; Zeller et al. 2018) or population connectivity (Wasserman et al. 2010). Therefore, the adoption of a spatial synoptic method incorporating ecological dispersal thresholds (sensu Cushman et al. 2014), such as the resistant kernel (Compton et al. 2007) or factorial least cost path (Cushman et al. 2009) approaches, provides a more powerful and useful means to identify and prioritise core areas, compared to a mere categorization of suitability surfaces (e.g., Macdonald et al. 2019; Li et al. 2022). Although inferences from resistant kernel modelling are highly sensitive to the threshold adopted (e.g., Riordan et al. 2016; Cushman et al. 2013b; Cushman and Landguth 2012), the results in this analysis highlighted that the importance of patches was generally related to their size (Figures 3 and 4), regardless of the dispersal threshold considered (Ahmadi et al. 2017), although their areas were weighted by the cumulative kernel intensity values inside those patches (e.g.; Kaszta et al. 2020a; Cushman et al. 2018) (Supplementary Tables S1 -S3). This importance is well reflected in the relative ranking of core patches within the network topology, for each of the indices considered (Figure 4). Larger patches generally ranked higher as they ensured increased intra-patch connection, more flux between patches, and they pillared the structural connectivity in the network (Figure 4; Supplementary Tables S1 and S2).

The shape, size and number of resistant kernel core areas varied with increasing dispersal threshold (Figure 3; Table 2), resulting in slightly different, but overlapping conclusions. One of the most important core habitat patches for snow leopard in the QLSNP landscape and neighbouring areas was located at the intersection between the counties of Subei, Sunan Yugur and Tianjun, on the north-western part (Figure 3). As reported in Atzeni et al. (2021), this area is important to functional connectivity, and is characterised by higher effective population sizes and higher genetic diversity, compared to neighbouring areas. In the centre, major core areas spanned across Qilian and Sunan Yugur Counties, or stretched till Tianjun and Delingha counties. In the south-eastern portion, the most important core area stretches over Menyuan, Tianzhu and Datong counties. At the largest thresholds, these patches progressively merged together highlighting four main backbone areas conducive of snow leopard large home-range movements (Figure 3).

These core areas present important analogies with previous connectivity models in snow leopard range. For example, they well match the $100 \mathrm{~km}$ resistant kernel patterns produced by Riordan et al. (2016). Riordan et al. (2016) also highlighted the prominent role of habitat patches located Delingha and Tianjun counties for range-wide landscape connectivity. In the same way, Li et al. (2020) identified the entirety of the Qilian mountains and neighbouring areas as a Landscape Conservation Unit, encompassing all the counties for which the resistant kernel values were found to be higher by the current analysis (Figure 3). Li et al. (2022) identified much extent of snow leopard suitable habitat outside the boundary of QLSNP, and highlighted four major conservation gaps located in the north-western, north central, and south portion of this landscape, confirming results from our kernel models (Figure 3). Likewise, Li et al. (2021) identified a conservation gap at the intersection of Qilian, Gangca and Tianjun counties, a critical area for snow leopard range-wide connectivity between the strongholds of Qilianshan and Sanjiangyuan National Parks (Li et al. 2021; Riordan et al. 2016).

The importance of all core patches identified in this analysis was confirmed by climatic models. Li et al. (2016) demonstrated that the Qilian mountains are a stable snow leopard refugium, that has not suffered from past climatic changes, and is likely to persist intact even under the worst climate change projections by 2070. These findings were confirmed by the recent assessment of Li et al. (2021), which also suggested a possible increase of snow leopard habitat inside QLSNP. All these independent pieces of evidence suggest that snow leopard populations are not confined to the Qilian mountains per se, and that a significant proportion of expected core areas is located in areas that functionally connect the north-eastern edge of the Qinghai-Tibetan Plateau, across Gansu and Qinghai, to its central parts in Qinghai province.

\subsection{Corridor importance}

This study identified hundreds of corridor fragments (Table 3), which were formally ranked on the basis of their strength, their length and the strength of the core areas they connected (Table 4) (Cushman et al. 2018; Kaszta et al. 2020a). Such ranking approaches are desirable to identify the locations on the landscape that may require intervention to mitigate possible detrimental land uses (Cushman et al. 2018; Khosravi et al. 2018; Kaszta et al. 2021). These corridors were generated through the factorial least-cost path algorithm, an approach that overcomes the limitations of classical least-cost path analyses (Cushman et al. 2009; 2013a), which constrain movement into narrow linear features not reflecting the species perception of the environment (Cushman et al. 2013a, b). Unlike analyses based on linear corridors

Page $11 / 24$ 
between patches, not including formal connectivity modelling (Diniz et al. 2017; Macdonald et al. 2019), these corridors are spatially-explicit and integrate the effects of landscape heterogeneity on movement, address path uncertainty through a smoothing procedure, and are able to represent specific population-level ecological processes at several dispersal capacities (Cushman et al. 2009; Cushman et al. 2013a).

Unlike core areas, there was not a direct relationship between size and corridor importance (Table 4). This provides further confirmation of the utility of such approach to prioritise potential corridors, and that not always longest paths are attributed the highest importance (Table 4). Common to both resistance scenarios, the most important corridors were often located outside the area delimited by QLSNP (Table 4; Figure 5), and this was particularly evident in the genetic scenario, where two long dispersal routes functionally connected the opposite extremes of the study area, through a series of movement adjacent to important habitat patches and to smaller stepping stones, ensuring range continuity (Figure 5). Notably, the two resistance scenarios identified important linkages connecting the southern-central portion either to the north-western and northern-central parts of the study landscape. Shortest links were instead representing intra-patch connectivity, or inter-patch connectivity along small fracture zones highlighted by the resistant kernels, either inside or outside the National Park (Figure 5).

Both scenarios, together with their integrated cumulative output (Figures 5 and 6) provide evidence of the continuity of a corridor network, which runs without interruption from QLSNP to neighbouring areas. Such corridors, especially those outside the National Park, are critical to ensure population viability, as they promote multi-generational gene flow, and possibly contrast the effects of isolation through genetic admixture (Christie and Knowles, 2015). Maintaining the integrity of such network would be the key to ensure the persistence of snow leopard populations on the north-eastern edge of the Qinghai-Tibetan Plateau and promote the preservation of important areas that are known to be fundamental for range-wide connectivity (Riordan et al. 2016; Li et al. 2020; Li et al. 2021; 2022).

\subsection{Comparison between resistance surfaces}

Our results showed that connectivity predictions inferred through a habitat-based resistance scenario resulted in very different patterns compared to those generated through a genetically optimised resistance surface, as exemplified by their low Pearson's correlation coefficients (Supplementary Figure S2). This provided further confirmation that these scenarios are based on different ecological processes, since one is consistent with factors maximizing fitness within home-ranges, and the other with landscape characteristics facilitating dispersal events and mating behaviour (Spear et al. 2010; Cushman et al. 2013a; Mateo-Sanchez et al. 2015a). Similar conclusions were reached by Mateo-Sanchez et al. $(2015 \mathrm{a}, \mathrm{b})$ in their study of connectivity of the brown bear in northern Spain, and by Wasserman et al. (2010) on American marten, a species, like the snow leopard, associated with high elevation montane ecosystems. The Mateo-Sanchez et al. (2015a) study assessed also differences in effective distances between the models, suggesting that a habitat-derived surface may tend to overestimate landscape resistances in areas characterized by low suitability.

Our models from QLSNP confirmed this observation. In fact, the habitat-based resistance scenario described major connectivity routes within core patches, whose direction outside core areas was governed by the patterns of suitability (Atzeni et al. 2020, Figure 5; Supplementary Figure S2). This is not surprising, as both core patches and habitat-based dispersal routes are derived from the same resistance scenario, reflecting the known relationships between snow leopards and landscape characteristics determining home-range selection (Atzeni et al. 2020). On the other hand, the genetic surface is largely dominated by the effect of fine scale ruggedness and most importantly elevation (Atzeni et al. in review), and identifies areas with flatter topographical characteristics within a suitable elevation range as the dominant locations conducive of gene flow (Figures 2 and 5; Supplementary Figure S2). The genetic resistance, however, largely overestimated effective distances within home range patches (Figure 5; Supplementary Figure S2). As noted by Mateo-Sanchez et al. (2015a), a genetic surface is obtained correlating pairwise genetic distances with landscape distances. Since any two individuals will experience a certain degree of genetic differentiation, animals located in close proximity, and likely in the same habitat core area, may be separated by high-cost distances.

Since the habitat-based corridors reflected the amount of suitable habitat, they resulted in more fragmented smaller links outside the extent delimited by the core areas. Specifically, their average length and area were consistently smaller than the pathways inferred by the genetic resistance, which were not constrained by habitat suitability, considering the whole extent (Table 3 ). The total extensiveness of the habitatderived paths was higher within the national park. As remarked above, both the core areas and the habitat-based resistance relied on the same surface, therefore the identification of a large number of patches inside the extent delimited by QLSNP resulted in a more marked fragmentation of smaller linkages (Tables 2 and 3 ).

Differences in connectivity patterns are common when comparing models generated through different kinds of data or different modelling approaches (Cushman and Lewis 2010; Cushman et al. 2014; Shirk et al. 2015; Zeller et al. 2018; Hearn et al. 2019). Some studies have shown that models relying on the same variables but assessed with different empirical dataset may retrieve the same connectivity routes (Cushman and Lewis 2010), or that models generated through different approaches and different variables might still reach a high degree

Page $12 / 24$ 
of concordance when describing movement patterns (Hearn et al. 2019). However, this is not always the case, as Cushman et al. (2014) have shown, for example, that two models with high concordance and based on the same variables yielded very different predictions of crossing localities for the American black bear, and Zeller et al. (2018), in an extensive comparison, demonstrated that even if resistance models built with different empirical data included always the same landscape constraints, they frequently predicted very different patterns of connectivity, with many of them unsupported with respect to a null model. Shirk et al. (2015), comparing an empirical derived resistance map to expert-based surfaces, reached the same conclusions on different connectivity patterns generated by the adoption of variables of the same nature.

With regards to the results of this study, besides considerations underlying the ecological phenomena described by the two data kinds, different patterns can also be attributed first to the adoption of a different suite of variables (Cushman and Lewis 2010; Hearn et al. 2019), and second to the extrapolation of results generated for a limited extent to a larger area (Cushman and Lewis 2010; Wasserman et al. $2012 b)$. In the first case, the two models, driven by different ecological assumptions, differed in the suite of predictors included, at topographical and landcover levels (Atzeni et al. 2020; Atzeni et al. in review). In the second case, the genetic resistance was derived using a portion of the landscape limited to Gansu province (Atzeni et al. 2021; Atzeni et al. in review), and its results greatly dependent on landscape characteristics pertinent to the north-western portion of the extent analysed in this study. Thus, in order to allow full comparability of connectivity patterns, future works should seek to adopt the same suite of predictors and to conduct inferences on the same extent (Cushman and Lewis 2010; Hearn et al. 2019), either related to home-range selection or drivers of gene flow.

Although in absolute terms previous evaluations have shown the superiority of genetically-derived resistance maps, compared to those based on habitat surrogates (Zeller et al. 2018), in light of the extent limitations pertinent to the genetic resistance map, it is not unrealistic to adopt an integrated connectivity map (Zeller et al. 2017, 2018) (Figure 6; Supplementary Figure S3), representing the magnitude of withinhome range movement paths, and the long-distance connectivity patterns following dispersal events related to mating behaviour and gene flow. The implementation of telemetry studies, currently ongoing and run by the Wildlife Institute of Beijing Forestry University, for the same extent covered by the QLSNP, will provide further insights on landscape constraints to movement and allow empirical validations of either scenario analysed in this study.

\section{Management Implications And Conclusions}

This analysis explored the effectiveness of the newly established QLSNP in ensuring landscape-level protection to snow leopard populations core habitat areas and connectivity routes. The evidence generated by this analysis however highlighted that the protected area alone might be inadequate to ensure complete protection of snow leopard landscapes (Tables 2 and 3 ), especially when this is set in a context of meta-population connectivity between distant parts of the Chinese range (Li et al. 2020, 2021, 2022). QLSNP, in fact, accounted only for $40 \%$ of the total extent covered by core habitat patches (Table 2). Cumulatively, QLSNP encompassed shorter and smaller corridor fragments, accounting for only $37-41 \%$ of total corridor length and a mere $30 \%$ of total corridor area (Table 3 ). Many of the strongest links were in fact located outside QLSNP, or functionally connected areas within the park to other localities not protected under any designation (Figs. 5 and 6).

The framework adopted has the advantage of providing a synoptic representation of connectivity patterns and core areas integrating two complementary approaches (e.g., Cushman et al. 2014). By providing an objective means to rank both core patches and factorial least-cost paths, it is possible to tailor conservation strategies on the basis of scientific evidence (Cushman et al. 2018; Kaszta et al. 2019, 2020a; Puyravaud et al. 2017). Many studies adopting such approaches have shown that, in the context of carnivore conservation, protected areas alone may fail to fully encompass the areas with higher expected movement probabilities (e.g., Kaszta et al. 2020a; Khosravi et al. 2018; Mohammadi et al. 2020) or provide protection to dispersal routes (e.g., Ashrafzadeh et al. 2020; Kaszta et al. 2019, 2020a). Identifying and protecting major core areas and the most important dispersal routes linking them, especially when focusing on large territorial carnivores, is a priority to ensure meta-population viability and to reduce the chances of isolation, with consequent deleterious demographic and genetic effects.

The adoption of the analytical tools employed in this analysis has elsewhere been incorporated into planning and implementation of new management initiatives. For example, the results from Cushman et al. (2018) have been used by the Government of Botswana to support conservation initiatives for the lion (Panthera leo) (Kaszta et al. 2020a). The evidence generated by Puyravaud et al. (2017) allowed the Indian government to suppress development plans along key Asian elephant (Elephas maximus) corridors (Kaszta et al. 2020a). In the same way, the results from this study might not only prompt further sampling efforts, but also guide management initiatives aimed at assessing new areas to be included under legal protection, encompassing both major corridors and core patches outside the extent of the current National Park (Li et al. 2021, 2022G). Given the undeniable importance of PAs for carnivore conservation (Elliot et al. 2014; Moqanaki and Cushman 2016, Ashrafzadeh et al. 2020; Khosravi et al. 2018), a valuable strategy to ensure adequate landscape protection would be the 
establishment of a network of PAs. This might have the positive effect to functionally connect the Qilianshan landscape to other important snow leopard strongholds on the Tibetan-Plateau, like Sanjiangyuan National Park (Li et al. 2021), which stands between the Landscape Conservation Units of Qilianshan and Hengduan (Li et al. 2020). Moreover, enhancing landscape protection for the snow leopard, along with mitigation of human activities and human-wildlife conflicts where applicable, might have beneficial effects to a plethora of species, among which wild ungulates, which are essential for the viability of healthy snow leopard populations.

These considerations suggest efforts to integrate the Qilian landscape and adjoining areas into National and International Plans aimed at identifying and protecting snow leopard core landscapes, stepping stone areas and corridor linkages among them, confirming the conclusion of previous studies of the same kind (Li et al. 2020, 2021, 2022a). The China Snow Leopard Conservation Action Plan (NFGA, 2013), among other objectives, proposed the establishment of new protected zones in areas without a management status, known to harbour snow leopard populations or to be important ecological corridors. Where snow leopards already occurred, the use of scientific evidence was advocated to promote evaluation of habitat quality, habitat restoration, restoration of prey populations, and the reconversion of farmland and grazing land to forests and grassland, respectively. The identification of key corridors from this analysis also supports the Qilianshan National Park Authorities' management initiatives, which foresaw the establishment of 3,000 wildlife corridors, mainly through fence removal, ecological restoration and mitigation of the effect of road and railway infrastructures (QLSNP Masterplan 2018). Since conservation efforts focus particularly on key flagship species, such the snow leopard and the white-lipped deer (Cervus albirostris), the results generated from this analysis will assist in the identification of localities where such management actions are more needed to ensure within-park connectivity.

At a global management level, the Global Snow Leopard \& Ecosystem Protection (GLSEP) initiative, a joint effort from snow leopard range countries to protect snow leopards and their habitats, sought to identify and prioritise the protection of at least 20 key landscapes possibly harbouring 100 individuals, by 2020 (GSLEP 2013). The results from this analysis suggest that the inclusion of the sole extent of Yanchiwan National Nature Reserve (GSLEP 2013), as foreseen by GSLEP agenda, would be not sufficient to ensure protection of snow leopards in this part of the range, or, critically, linkage of this part of the Chinese snow leopard range with other key habitat areas. Based on the estimates from of one individual per $100 \mathrm{~km}^{2}$ from Li et al. (2020), the analysis of the core habitat patches would support 187-200 individuals within the sole extent of QLSNP, and possibly as much as 471 considering the adjoining areas not included within the park.

In conclusion, this study is the first example to have applied two complementary synoptic methods of spatial connectivity at landscape-level on snow leopard. This differs from previous range-wide and local studies (Riordan et al. 2016; Hameed et al. 2020; Shrestha and Kindlmann 2020) in that it provided explicit ranking of major core habitat areas and the spatial location of the most important stepping-stones and movement corridors. While some methodological issues exist, especially with regard to the extrapolation of the genetic resistance to a broader area, the results in either scenario analysed encompass exclusively abiotic landscape constraints. Future improvements might seek to incorporate direct and indirect threats posed by anthropic factors, represented by activities, transportation infrastructure (Kaszta et al. 2020b; Li et al. 2022), human population, grazing pressure, and may also consider to include information on natural prey populations, which are an essential factor for the persistence of viable populations of snow leopards. Additionally, it would be informative to evaluate climate change scenarios on core areas and connectivity for snow leopards with this analytical framework, as has been done, for example, for American marten (Wasserman et al. 2012a, b). As discussed previously, it will also be essential to validate both scenarios through the analysis of telemetry data, which give enhanced power to capture factors involved in dispersal processes (Zeller et al. 2017, 2018; see Johansson et al. 2021).

\section{Declarations}

National Natural Science Foundation of China (Grant No. 31470567)

\section{Competing Interest}

All the authors declare no competing interests involved with this manuscript.

\section{Author Contributions}

LA, SK, PR and SC conceived the study. LA, PR, SK, and SC supervised modeling analyses. LA and WJ conducted modeling analyses. LA wrote the manuscript. All authors provided contributions through comments and editing of the final version.

\section{Data Availability}


The distribution model and resistance surfaces adopted in this study are considered sensitive data by National Forestry and Grassland Administration of China (NFGA). As such, limitations on the free distribution of such data apply. The corresponding author welcomes inquiry about the use of our resistance and distribution models in related frameworks focusing on snow leopard conservation in the Qilianshan landscape.

\section{Acknowledgments}

This manuscript utilised model outputs generated through analyses of empirical data pertinent to snow leopard occurrences and genetic structure. The authors acknowledge support from the Second National Survey of Terrestrial Wildlife in China, National Forestry and Grassland Administration of China (NFGA), and National Natural Science Foundation of China (Grant No. 31470567). We thank the Yanchiwan National Nature Reserve authorities, especially Mr. Wuliji, Mr. Dazhan, Mr. Wan Shengqi, Mr. Dou Zhigang, and Mr. Pei Wen, for their help and support with the fieldwork in Gansu Province, China. We thank the Gansu Qilianshan National Nature Reserve authorities, especially Mr. Ma Duifeng and Mr. Liao Kongtai for their support with fieldwork. We further thank the Qinghai Province Qilianshan Nature Reserve authorities, in particular Ms. Gao Yayue, Mr. Zhang Yu, and Mr. Han Qiang for providing help and support with data collection. Luciano Atzeni warmly thanks Mr. Andrea Ragatzu, for graphical improvements on the figures presented in this manuscript.

\section{References}

1. Adriansen F, Chardon JP, De Blust G, Swinnen E, Gulinck H, and Matthysen E. The application of "least-cost" modelling as a functional landscape model. Landsc Urb Plann. (2003) 64, 223-47.

2. Ahmadi M, Nezami Balouchi B, Jowkar H, Hemami MR, Fadakar D, Malakouti-Khah S, and Ostrowski S. Combining landscape suitability and habitat connectivity to conserve the last surviving population of cheetah in Asia. Divers Distrib. (2017) 23, 592-603

3. Alexander JS, Cusack JJ, Pengju C, Kun S, and Riordan P. Conservation of snow leopards: spill-over benefits for other carnivores?. Oryx. (2015) 1-5.

4. Ashrafzadeh MR, Khosravi R, Adibi MA, Taktehrani A, Wan HY, and Cushman SA. A multi-scale, multi-species approach for assessing effectiveness of habitat and connectivity conservation for endangered felids. Biol Conserv. (2020) 245, 108523.

5. Atzeni L, Cushman SA, Bai D, Wang J, Chen P, Shi K, and Riordan P. Meta-replication, sampling bias, and multi-scale model selection: A case study on snow leopard (Panthera uncia) in western China. Ecol Evol. (2020).

6. Atzeni, L., Cushman, S.A., Wang, J., Riordan, P., Shi, K. \& Bauman, D. (2021). Evidence of spatial genetic structure in a snow leopard population from Gansu, China. Heredity 1-13.

7. Christie MR, and Knowles LL. Habitat corridors facilitate genetic resilience irrespective of species dispersal abilities or population sizes. Evol Appl. (2015) 8, 454-463.

8. Compton BW, Mcgarigal K, Cushman SA, and Gamble LR. A Resistant-Kernel Model of Connectivity for Amphibians that Breed in Vernal Pools. Conserv Biol. (2007) 21, 788-799.

9. Cushman S, McKelvey KS, Hayden J, and Schwartz M. Gene Flow in Complex Landscapes: Testing Multiple Hypotheses with Causal modelling. Am Nat. (2006) 168, 486-499.

10. Cushman SA, and Landguth EL. Ecological associations, dispersal ability, and landscape connectivity in the northern Rocky Mountains. Res. Pap. RMRS-RP-90. Fort Collins, CO: U.S. Department of Agriculture, Forest Service, Rocky Mountain Research Station. (2012a). 21

p.

11. Cushman SA, and Landguth EL. Ecological associations, dispersal ability, and landscape connectivity in the northern Rocky Mountains. Res. Pap. RMRS-RP-90. Fort Collins, CO: U.S. Department of Agriculture, Forest Service, Rocky Mountain Research Station. (2012). 21 p.

12. Cushman SA, and Landguth EL. Scale dependent inference in landscape genetics. Landsc Ecol. (2010) 25, 967-979.

13. Cushman SA, and Lewis JS. Movement behaviour explains genetic differentiation in American black bears. Landsc Ecol. (2010) 25, 1613-1625.

14. Cushman SA, Elliot NB, Bauer D, ... Loveridge AJ. Prioritizing core areas, corridors and conflict hotspots for lion conservation in southern Africa.. PLoS One. (2018) 13, e0196213.

15. Cushman SA, Landguth EL, and Flather $\mathrm{CH}$. Evaluating population connectivity for species of conservation concern in the American Great Plains. Biodivers Conserv. (2013b) 22, 2583-2605.

16. Cushman SA, Landguth $\mathrm{EL}$, and Flather $\mathrm{CH}$. Evaluating the sufficiency of protected lands for maintaining wildlife population connectivity in the U.S. northern Rocky Mountains. Divers Distrib. (2012b) 18, 873-884. 
17. Cushman SA, Lewis JS, and Landguth EL. Why did the bear cross the road? Comparing the performance of multiple resistance surfaces and connectivity modelling methods. Diversity. (2014) 6, 844-854.

18. Cushman SA, McKelvey KS, and Schwartz MK. Use of Empirically Derived Source-Destination Models to Map Regional Conservation Corridors. Conserv Biol. (2009) 23, 368-376.

19. Cushman SA, McRae B, Adriaensen F, Beier P, Shirley M, and Zeller KA. Biological corridors and connectivity. In: Key topics in conservation biology 2 ed. D. MacDonald D, and Willis K. (2013a) pp. 384-404.

20. Cushman SA, Raphael MG, Ruggiero LF, Shirk AS, Wasserman TN, and O'Doherty EC. Limiting factors and landscape connectivity: the American marten in the Rocky Mountains. Landsc Ecol. (2011) 26, 1137-1149.

21. Cushman SA, Shirk AJ, and Landguth EL. Landscape genetics and limiting factors. Conserv Genet. (2013c) 14, $263-274$.

22. CUSHMAN, S.A., McKELVEY, K.S. \& SCHWARTZ, M.K. (2009). Use of Empirically Derived Source-Destination Models to Map Regional Conservation Corridors. Conservation Biology 23, 368-376.

23. Dijkstra EW. A note on two problems in connection with graphs. Numerische Mathematik. (1959) 1, $269-271$.

24. Diniz MF, Cushman SA, Machado RB, and Júnior PDM. Landscape connectivity modelling from the perspective of animal dispersal. Landsc Ecol. (2020) 35, 41-58.

25. Diniz MF, Machado RB, Bispo AA, and Brito D. Identifying key sites for connecting jaguar populations in the Brazilian Atlantic Forest. Anim Conserv. (2017) 21, 201-210.

26. Elliot NB, Cushman SA, Macdonald DW, and Loveridge AJ. The devil is in the dispersers: predictions of landscape connectivity change with demography. J Appl Ecol. (2014) 51, 1169-1178.

27. Evans JS. spatialEco. R package version 1.3-1. (2020) URL https://github.com/jeffreyevans/spatialEco.

28. Fahrig L. Effects of habitat fragmentation on biodiversity. Ecol Evol Syst. (2003) 34, 487-515.

29. Ferrari JR, Lookingbill TR, and Neel MC. Two measures of landscape-graph connectivity: assessment across gradients in area and configuration. Landsc Ecol. (2007) 22, 1315-1323.

30. GSLEP. Global Snow Leopard and Ecosystem Protection Program. (2013) Bishkek, Kyrgyz Republic: Snow Leopard Secretariat.

31. Hameed S, Din J ud, Ali H, ... Nawaz MA. Identifying priority landscapes for conservation of snow leopards in Pakistan. PLoS One. (2020) 15, e0228832.

32. Hearn AJ, Cushman SA, Goossens B, Ross J, Macdonald EA, Hunter LTB, and Macdonald DW. Predicting connectivity, population size and genetic diversity of Sunda clouded leopards across Sabah, Borneo. Landsc Ecol. (2019) 34, $275-290$.

33. Johansson Ö, Ausilio G, Low M, Lkhagvajav P, Weckworth B, and Sharma K. The timing of breeding and independence for snow leopard females and their cubs. Mamm Biol. (2021) 101, 173-180.

34. Johansson Ö, Koehler G, Rauset GR, Samelius G, Andrén H, Mishra C, Lhagvasuren P, McCarthy T, and Low M. Sex-specific seasonal variation in puma and snow leopard home range utilization. Ecosphere. (2018) 9, e02371.

35. Johansson Ö, Rauset G, Samelius G, Tom M, Andrén H, Tumursukh L, and Mishra C. Land sharing is essential for snow leopard conservation. Biol Conserv. (2016) 203, 1-7.

36. Kaszta Ż, Cushman SA, and Macdonald DW. Prioritizing habitat core areas and corridors for a large carnivore across its range. Anim Conserv. (2020a) 23, 607-616.

37. Kaszta Ż, Cushman SA, Hearn AJ, Burnham D, Macdonald EA, Goossens B, Nathan SKSS, and Macdonald DW. Integrating Sunda clouded leopard (Neofelis diardi) conservation into development and restoration planning in Sabah (Borneo). Biol Conserv. (2019) 235, 63-76.

38. Kaszta Ż, Cushman SA, Htun S, Naing H, Burnham D, and Macdonald DW. Simulating the impact of Belt and Road initiative and other major developments in Myanmar on an ambassador felid, the clouded leopard, Neofelis nebulosa. Landsc Ecol. (2020b) 35, 727-746.

39. Keeley ATH, Beier P, and Gagnon JW. Estimating landscape resistance from habitat suitability: effects of data source and nonlinearities. Landsc Ecol. (2016) 31, 2151-2162.

40. Keeley ATH, Beier P, Keeley BW, and Fagan ME. Habitat suitability is a poor proxy for landscape connectivity during dispersal and mating movements. Landsc Urban Plan. (2017) 161, 90-102.

41. Khosravi R, Hemami M, and Cushman SA. Multispecies assessment of core areas and connectivity of desert carnivores in central Iran. Divers Distrib. (2018) 24, 193-207.

42. Landguth EL, Hand BK, Glassy J, Cushman SA, and Sawaya MA. UNICOR: A species connectivity and corridor network simulator. Ecography (Cop). (2012) 35, 9-14. 
43. Li J, Mccarthy TM, Wang H, Weckworth B V, Schaller GB, Mishra C, Lu Z, and Beissinger SR. Climate refugia of snow leopards in High Asia. BIOC. (2016) 203, 188-196.

44. Li J, Weckworth B V, McCarthy TM, ... Beissinger SR. Defining priorities for global snow leopard conservation landscapes. Biol Conserv. (2020) 241, 108387.

45. Li, J., Xue, Y., Hacker, C.E., Zhang, Y., Li, Y., Cong, W., Jin, L., Li, G., Wu, B., Li, D. \& Zhang, Y. (2021). Projected impacts of climate change on snow leopard habitat in Qinghai Province, China. Ecol Evol.

46. Li, Y.; Zhang, Y.; Xue, Y.; Zhang, Y.; Zhang, Y.; Gao, Y.; Li, D. Analysis of Conservation Gaps and Landscape Connectivity for Snow Leopard in Qilian Mountains of China. Sustainability 2022, 14, 1638. https://doi.org/10.3390/ su14031638

47. Macdonald DW, Bothwell HM, Kaszta Ż, et al (2019) Multi-scale habitat modelling identifies spatial conservation priorities for mainland clouded leopards (Neofelis nebulosa). Divers Distrib 25:1639-1654. https://doi.org/10.1111/ddi.12967

48. Macdonald DW, Chiaverini L, Bothwell HM, ... Cushman SA. Predicting biodiversity richness in rapidly changing landscapes: climate, low human pressure or protection as salvation?. Biodivers Conserv. (2020) 29, 4035-4057.

49. Macdonald EA, Cushman SA, Landguth EL, Hearn AJ, Malhi Y, and Macdonald DW. Simulating impacts of rapid forest loss on population size, connectivity and genetic diversity of Sunda clouded leopards (Neofelis diardi) in Borneo. PLoS One. (2018) 13, e0196974.

50. Mateo-Sánchez MC, Balkenhol N, Cushman S, Pérez T, Domínguez A, and Saura S. A comparative framework to infer landscape effects on population genetic structure: are habitat suitability models effective in explaining gene flow?. Landsc Ecol. (2015b) 1-16.

51. Mateo-Sánchez, M.C., Balkenhol, N., Cushman, S., Pérez, T., Domínguez, A. \& Saura, S. (2015a). Estimating effective landscape distances and movement corridors: comparison of habitat and genetic data. Ecosphere 6, 1-16.

52. McCarthy T, Mallon D, Jackson R, Zahler P, and McCarthy K. Panthera uncia. The IUCN Red List of Threatened Species 2017: e.T22732A50664030. [WWW Document]. (2017) URL http://dx.doi.org/10.2305/IUCN.UK.2017-2.RLTS.T22732A50664030.en.

53. McCarthy TM, Fuller TK, and Munkhtsog B. Movements and activities of snow leopards in Southwestern Mongolia. Biol Conserv. (2005) $124,527-537$.

54. McGarigal K, Cushman SA, and Ene E. FRAGSTATS v4: Spatial pattern analysis program for categorical and continuous maps. Computer software program produced by the authors at the University of Massachusetts, Amherst. (2012) URL http://www.umass.edu/landeco/research/fragstats/fragstats.html

55. McRae BH, Dickson BG, Keitt TH, and Shah VB. Using circuit theory to model connectivity in ecology, evolution, and conservation. Ecology. (2008) 89, 2712-2724.

56. Mohammadi A, Almasieh K, Nayeri D, Ataei F, Khani A, López-Bao J V, Penteriani V, and Cushman SA. Identifying priority core habitats and corridors for effective conservation of brown bears in Iran. Sci Rep. (2021) 11, 1044.

57. Moqanaki EM, and Cushman SA. All roads lead to Iran: predicting landscape connectivity of the last stronghold for the critically endangered Asiatic cheetah. Anim. Conserv. (2017) 20, 29-41.

58. NFGA. China Snow leopard Conservation Action Plan. National Forestry and Grassland Administration of China. (2013). (in Chinese).

59. Pascual-Hortal L, and Saura S. Comparison and development of new graph-based landscape connectivity indices: towards the priorization of habitat patches and corridors for conservation. Landsc Ecol. (2006) 21, 959-967.

60. Puyravaud J -P., Cushman SA, Davidar P, and Madappa D. Predicting landscape connectivity for the Asian elephant in its largest remaining subpopulation. Anim Conserv. (2017) 20, 225-234.

61. QLSNP Masterplan. Qilian Shan National Park Masterplan [WWW Document]. (2018) http://www.forestry.gov.cn/qls/3/index.html (in Chinese).

62. Riordan P, Cushman SA, Mallon D, Shi K, and Hughes J. Predicting global population connectivity and targeting conservation action for snow leopard across its range. (2016) 419-426.

63. Robinson HS, and Weckworth B. Landscape Ecology: Linking Landscape Metrics to Ecological Processes. In: Snow Leopards. Biodiversity of the world: from genes to landscapes ed. McCarthy T, Mallon D, and Nyhus P. (2016) pp. $395-405$.

64. Rudnick D, Ryan SJ, Beier P, Cushman SA, Dieffenbach F, and Trombulak SC. The role of landscape connectivity in planning and implementing conservation and restoration priorities. Issues Ecol. (2012) 16, 1-20.

65. Saura S, and Pascual-Hortal L. A new habitat availability index to integrate connectivity in landscape conservation planning: Comparison with existing indices and application to a case study. Landsc Urban Plan. (2007) 83, 91-103.

66. Saura S, and Rubio L. A common currency for the different ways in which patches and links can contribute to habitat availability and connectivity in the landscape. Ecography (Cop). (2010) 33, 523-537.

Page $17 / 24$ 
67. Saura S, and Torné J. Conefor Sensinode 2.2: A software package for quantifying the importance of habitat patches for landscape connectivity. Environ Model Softw. (2009) 24, 135-139.

68. Shirk AJ, Schroeder MA, Robb LA, and Cushman SA. Empirical validation of landscape resistance models: insights from the Greater Sage-Grouse (Centrocercus urophasianus). Landsc Ecol. (2015) 30, 1837-1850.

69. Shrestha B, and Kindlmann P. Implications of landscape genetics and connectivity of snow leopard in the Nepalese Himalayas for its conservation. Sci Rep. (2020) 10, 19853.

70. Spear SF, Balkenhol N, Fortin MJ, McRae BH, and Scribner K. Use of resistance surfaces for landscape genetic studies: Considerations for parameterization and analysis. Mol Ecol. (2010) 19, 3576-3591.

71. Suryawanshi KR, Khanyari M, Sharma K, Lkhagvajav P, and Mishra C. Sampling bias in snow leopard population estimation studies. Popul Ecol. (2019) 61, 268-276.

72. Taylor PD, Fahrig L, Henein K, and Merriam G. Connectivity is a vital element of landscape structure. Oikos. (1993). 68, $571-573$.

73. Urban D, and Keitt T. Landscape connectivity: a graph-theoretic perspective. Ecology. (2001) 82, 1205-18.

74. Wasserman TN, Cushman SA, Littell JS, Shirk AJ, and Landguth EL. Population connectivity and genetic diversity of American marten (Martes americana) in the United States northern Rocky Mountains in a climate change context. Conserv Genet. (2012a) 14, $529-541$.

75. Wasserman TN, Cushman SA, Schwartz MK, and Wallin DO. Spatial scaling and multi-model inference in landscape genetics: Martes americana in northern Idaho. Landsc Ecol. (2010) 25, 1601-1612.

76. Wasserman TN, Cushman SA, Shirk AS, Landguth EL, and Littell JS. Simulating the effects of climate change on population connectivity of American marten (Martes americana) in the northern Rocky Mountains, USA. Landsc Ecol. (2012b) 27, $211-225$.

77. Weckworth B. Snow Leopard (Panthera uncia) Genetics: The Knowledge Gaps, Needs, and Implications for Conservation. J Indian Inst Sci. (2021) 1-12

78. Wiens JA. The landscape context of dispersal. In: Dispersal ed. Clobert J, Danchin E, Dhondt AA, and Nichols JD. (2001) pp. 96-109.

79. Zeller KA, Jennings MK, Vickers TW, Ernest HB, Cushman SA, and Boyce WM. Are all data types and connectivity models created equal? Validating common connectivity approaches with dispersal data. Divers Distrib. (2018) 24, 868-879.

80. Zeller KA, McGarigal K, and Whiteley AR. Estimating landscape resistance to movement: a review. Landsc Ecol. (2012) $27,777-797$.

81. Zeller KA, Vickers TW, Ernest HB, and Boyce WM. Multi-level, multi-scale resource selection functions and resistance surfaces for conservation planning: Pumas as a case study. PLoS One. (2017) 12: e0179570.

\section{Figures}




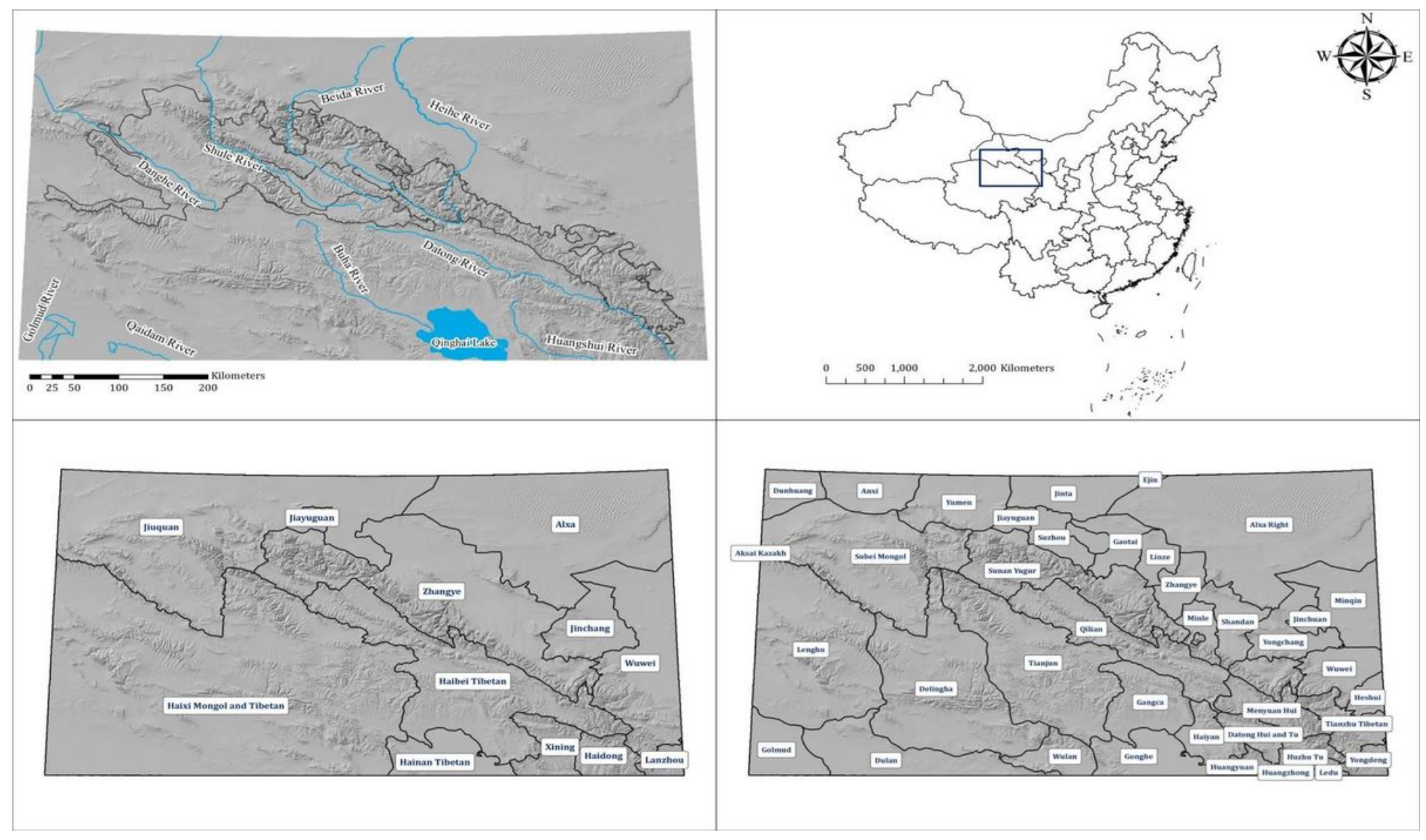

Figure 1

Overview of the study area. Top left: Major rivers and lakes in the area; borders represent Qilianshan National Park (QLSNP). Top right: location of the studied extent within China. Bottom left: prefecture-level administrative divisions of the area. Bottom right: county-level administrative divisions of the area 


\section{A) Genetic Resistance}
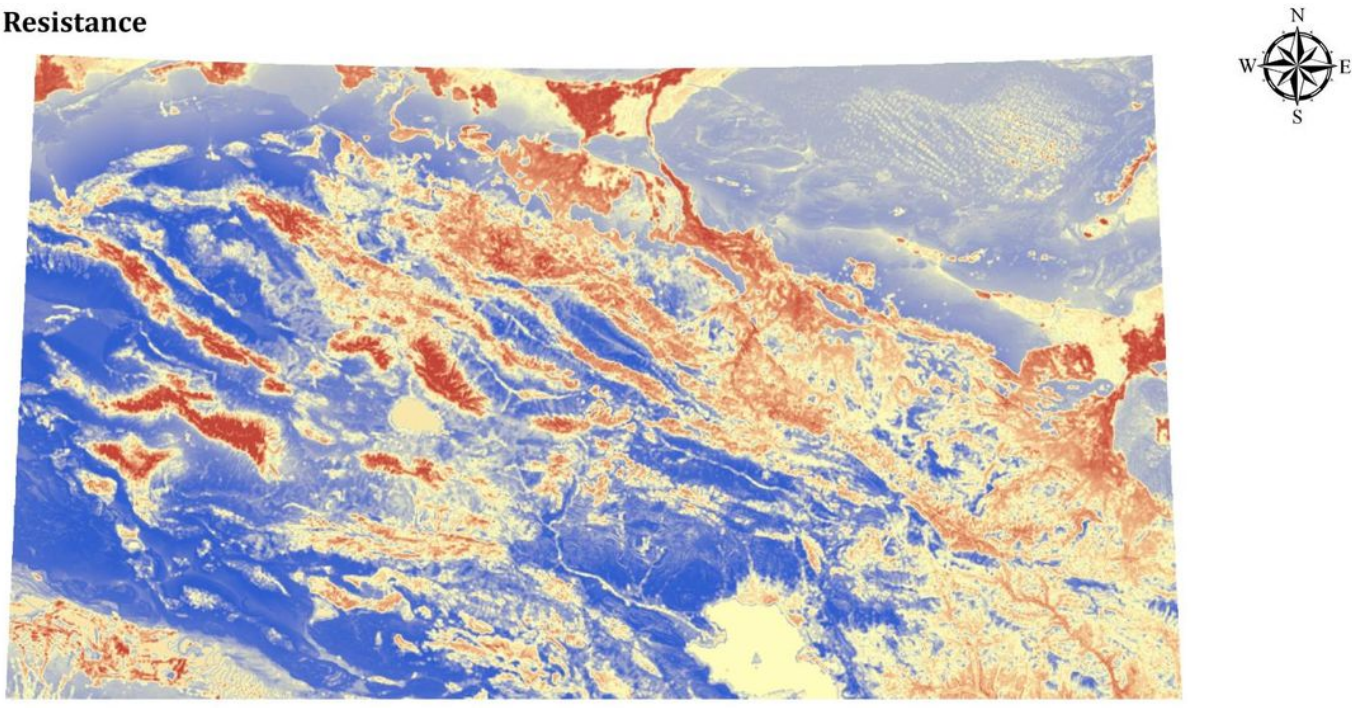

\section{B) Habitat-based Resistance}

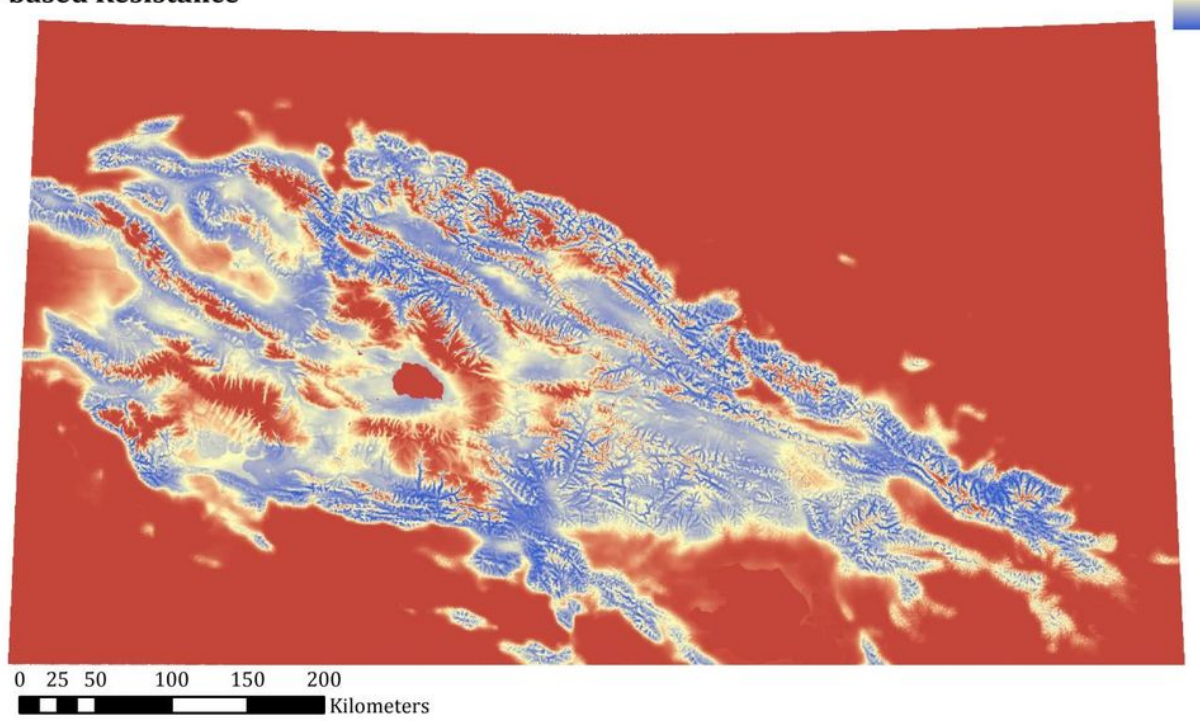

\section{Resistance}

\section{Figure 2}

Resistance surfaces adopted in this analysis. Genetic resistance (A), and Habitat-based resistance (B) 


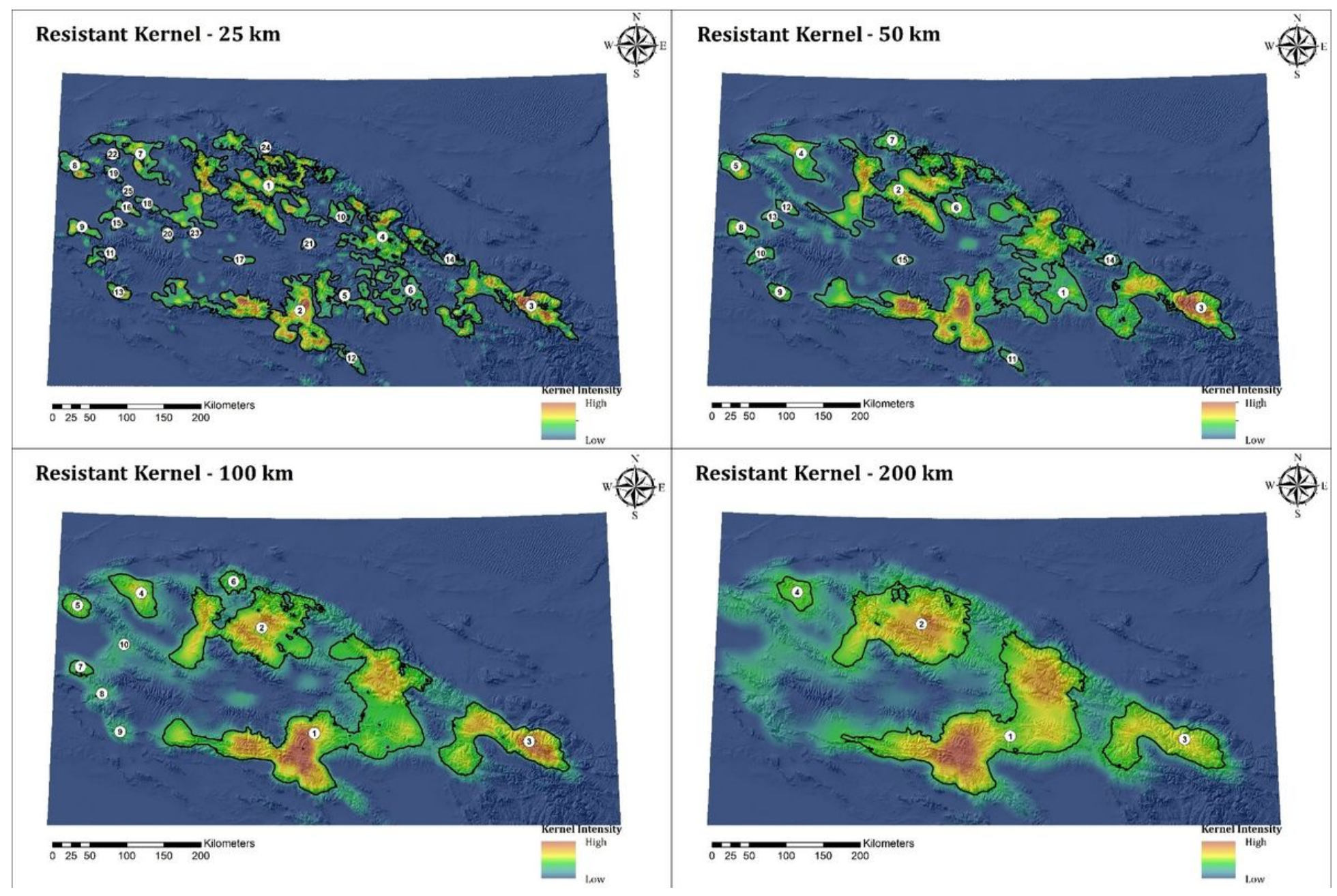

Figure 3

Resistant kernel core area patches at all the thresholds (expressed in km-equivalent units) considered 


\section{Resistant kernel $25 \mathrm{~km}$ core patches}

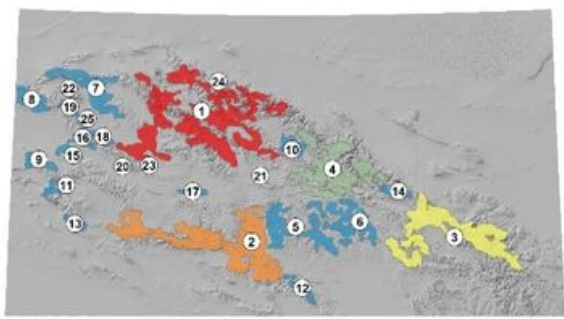

\begin{tabular}{r|l} 
Intra & $\square .647527-2.729270$ \\
\hline $0.000000-0.041297$ & $2.729271-6.757840$ \\
\hline $0.041298-0.647526$ & $6.757841-21.234100$
\end{tabular}
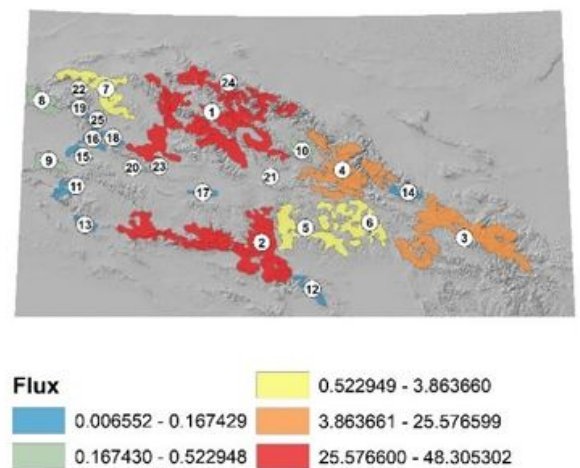

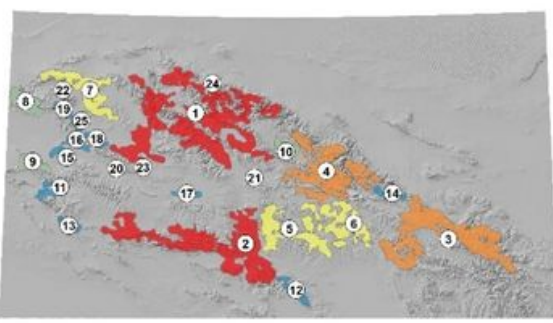

Connector $0.522949-3.863660$

\begin{tabular}{|c|c|c|c|}
\hline $0.006552-0.167429$ & $3.863661-25.576599$ \\
\hline
\end{tabular} $0.167430-0.522948 \square 25.576600-48.305302$

\section{Resistant kernel 50 km core patches}
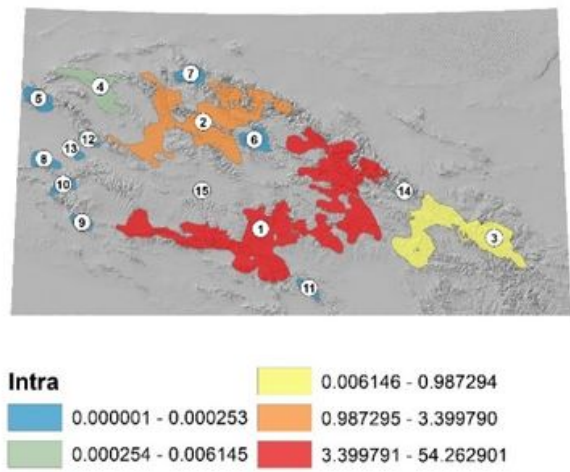
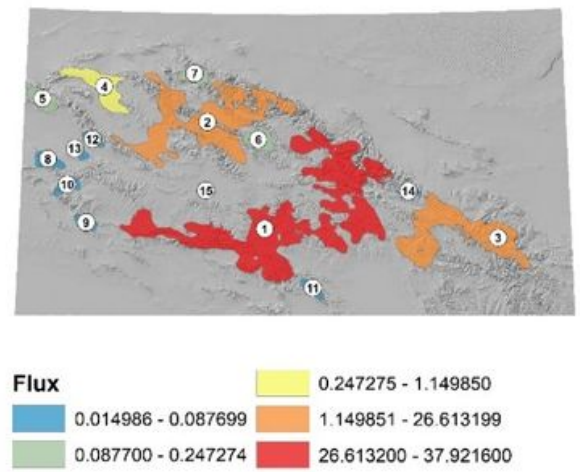

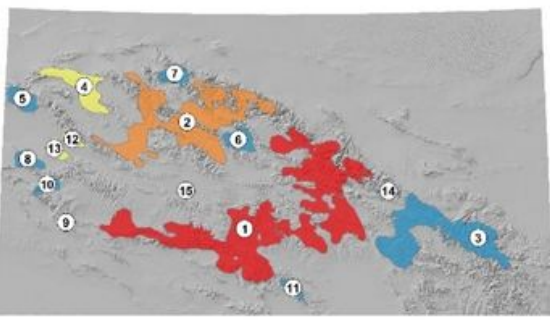

\begin{tabular}{|c|l|} 
Connector & $0.000600-0.016220$ \\
\hline $0.000000-0.000007$ & $0.016221-0.606799$ \\
\hline $0.000008-0.000599$ & $0.606800-2.014360$ \\
\hline
\end{tabular}

Figure 4

Values of the Intra, Flux and Connector fractions of the probability of connectivity (PC) index for the kernel core patches at 25 and $50 \mathrm{~km}$ equivalent 


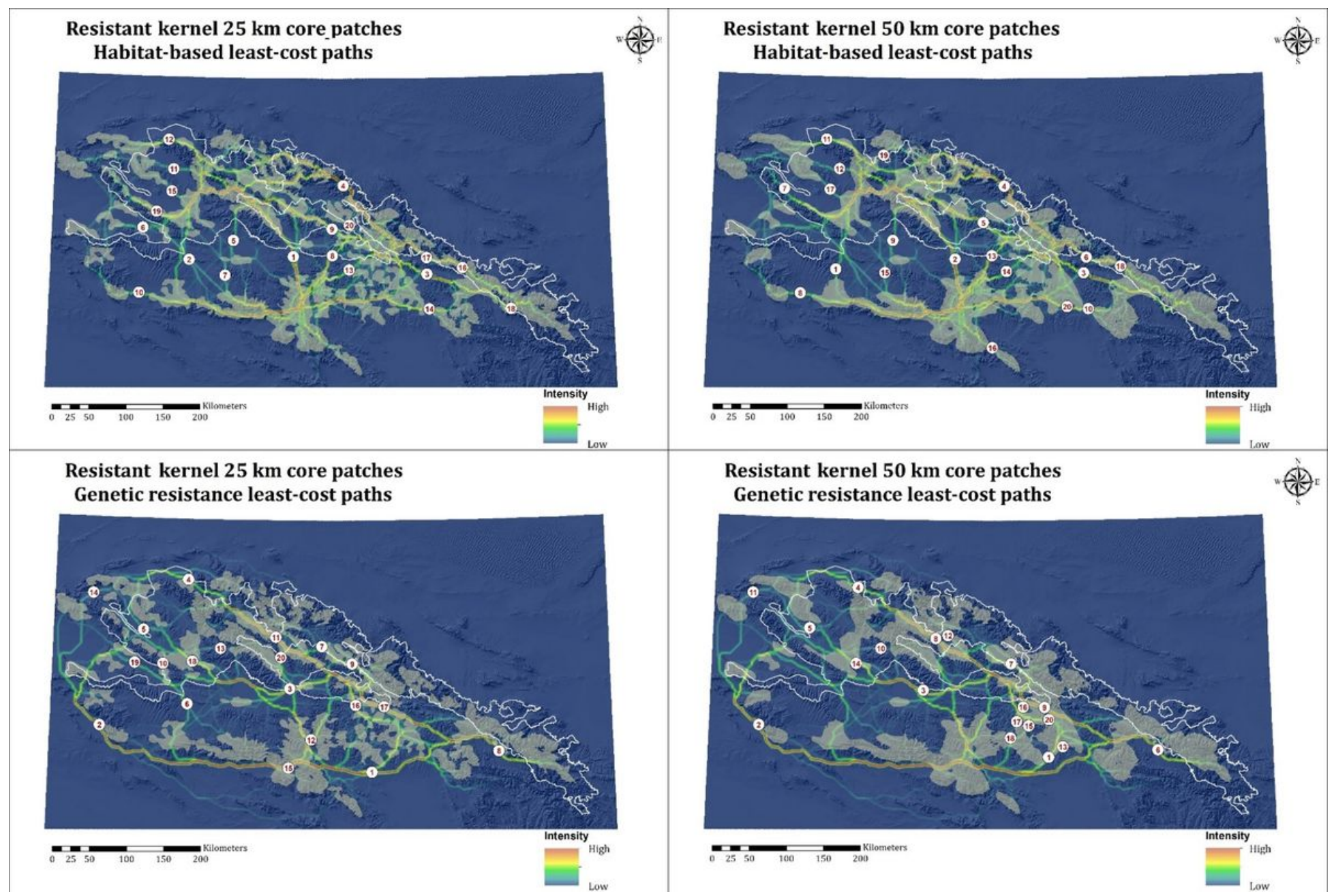

Figure 5

Location of the 20 most important factorial least-cost path corridors connecting the core habitat patches at 25 and $50 \mathrm{~km}$ equivalent in the two resistance scenarios 


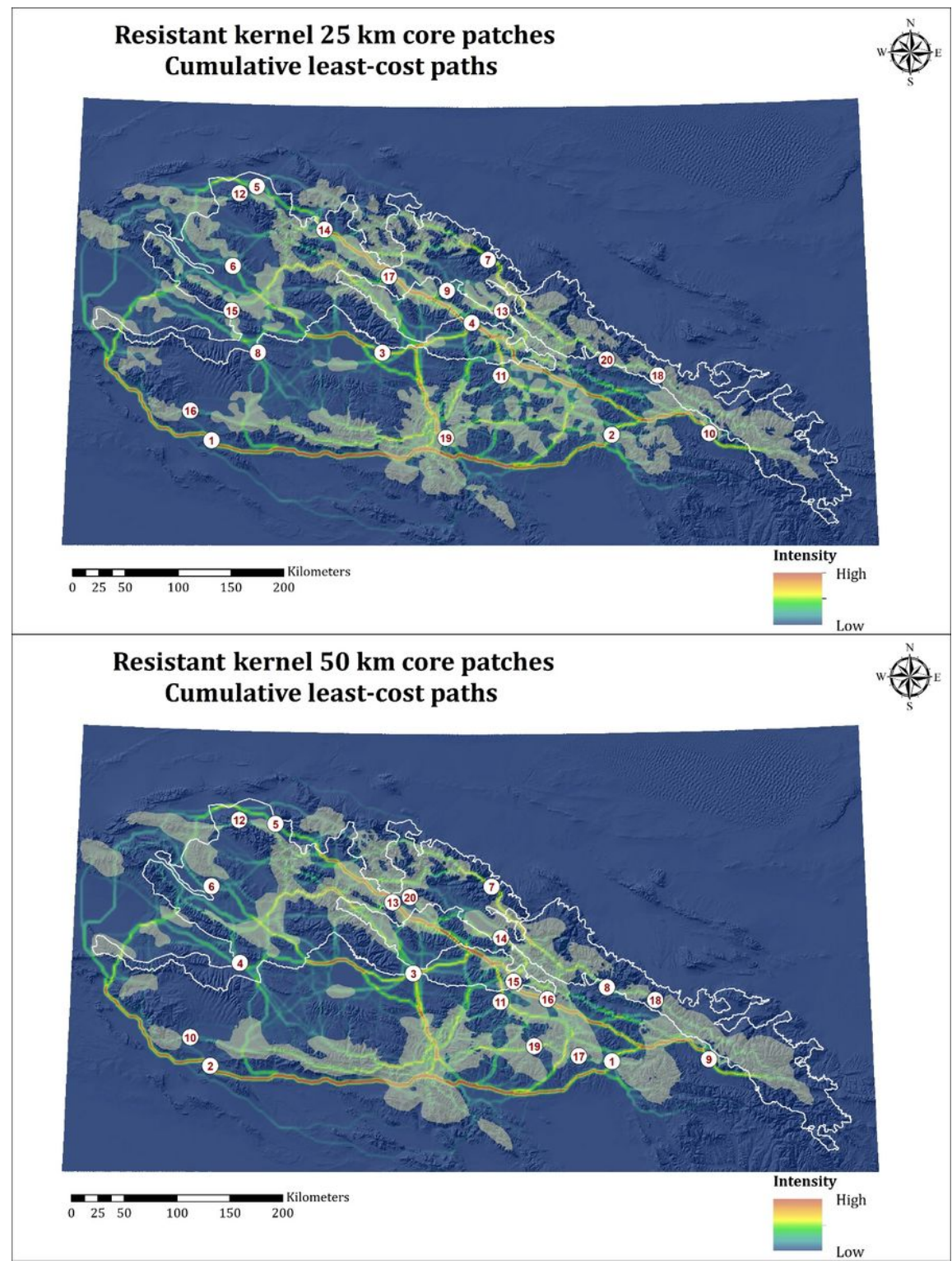

\section{Figure 6}

Location of the 20 most important corridors connecting the core habitat patches at 25 and 50 km equivalent. The corridors were identified from the cumulative outputs of the factorial least-cost paths calculated from the habitat-based and genetic-based resistance scenarios

\section{Supplementary Files}

This is a list of supplementary files associated with this preprint. Click to download.

- Supplementary.pdf 